\title{
Sufficient conditions for the existence of periodic solutions of the extended Duffing-Van der Pol oscillator
}

Rodrigo D. Euzébio \& Jaume Llibre

To cite this article: Rodrigo D. Euzébio \& Jaume Llibre (2016) Sufficient conditions for the existence of periodic solutions of the extended Duffing-Van der Pol oscillator, International Journal of Computer Mathematics, 93:8, 1358-1382, DOI: 10.1080/00207160.2015.1046847

To link to this article: https://doi.org/10.1080/00207160.2015.1046847

Accepted author version posted online: 30

Apr 2015.

Published online: 09 Jun 2015.

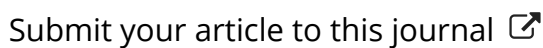

凹ll Article views: 90

View Crossmark data $\complement$ 


\title{
Sufficient conditions for the existence of periodic solutions of the extended Duffing-Van der Pol oscillator
}

\author{
Rodrigo D. Euzébio ${ }^{\mathrm{a}, \mathrm{b}}$ and Jaume Llibre ${ }^{\mathrm{a} *}$ \\ ${ }^{a}$ Departament de Matemàtiques, Universitat Autònoma de Barcelona, 08193 Bellaterra, Barcelona, \\ Catalonia, Spain, ${ }^{b}$ Departament de Matemática, IBILCE, UNESP, Rua Cristovao Colombo 2265, Jardim \\ Nazareth, CEP 15.054-00, Sao José de Rio Preto, SP, Brazil
}

(Received 20 October 2014; revised version received 17 March 2015; accepted 28 March 2015)

\begin{abstract}
In this paper, some aspects on the periodic solutions of the extended Duffing-Van der Pol oscillator are discussed. Doing different rescaling of the variables and parameters of the system associated with the extended Duffing-Van der Pol oscillator, we show that it can bifurcate one or three periodic solutions from a two-dimensional manifold filled by periodic solutions of the referred system. For each rescaling we exhibit concrete values for which these bounds are reached. Beyond that we characterize the stability of some periodic solutions. Our approach is analytical and the results are obtained using the averaging theory and some algebraic techniques.
\end{abstract}

Keywords: extended Duffing-Van der Pol oscillator; periodic solution; non-autonomous systems; averaging theory

2010 AMS Subject Classifications: Primary: 34C07; 34C15; 34C25; 34C29; 37C60

\section{Introduction}

\subsection{Setting the problem}

A large number of non-autonomous chaotic phenomena in physics, engineering, mechanics and biology, among others, are described by second-order differential systems of the form

$$
\ddot{x}=g(x, \dot{x}, t)+\gamma(t),
$$

where $g(x, \dot{x}, t)$ is a continuous function and $\gamma(t)$ is some external force. For instance, in biology, system (1) models the FHN neuron oscillator, and in engineering, system (1) is a model to the horizontal platform system.

The specific topic addressed in this paper concerns another particular case of Equation (1), namely, an extension of the forced Van der Pol equation with external excitation. Van der Pol's system also plays an important role in many applications in areas such as engineering, biology, physics and seismology (see [2] and references therein).

*Corresponding author. Email: jllibre@mat.uab.cat 
The system associated with the forced Van der Pol equation with external excitation is characterized by system (1) with functions $g$ and $\gamma$ in the form

$$
g(x, \dot{x}, t)=\rho_{0}\left(1-x^{2}\right) \dot{x}-\frac{\mathrm{d}}{\mathrm{d} x} V(x), \quad \gamma(t)=\delta_{0} \cos (\omega t),
$$

where $\rho_{0}$ is the damping parameter, $V(x)$ is the potential function, and $\delta_{0}$ and $\omega$ are the amplitude and angular frequency of the driving force $\gamma(t)$, respectively. We assume that $\rho_{0}$ is non-negative and $\delta_{0}$ and $\omega$ are positive. The potential $V(x)$ can be approximated by a finite Taylor expansion in series

$$
V_{2}(x)=\frac{1}{2} \omega_{0}^{2} x^{2}, \quad V_{4}(x)=\frac{1}{2} \omega_{0}^{2} x^{2}+\frac{1}{4} \alpha_{0} x^{4}, \quad V_{6}(x)=\frac{1}{2} \omega_{0}^{2} x^{2}+\frac{1}{4} \alpha_{0} x^{4}+\frac{1}{6} \lambda_{0} x^{6},
$$

where $\omega_{0}$ and $\lambda_{0}$ are non-zero and $\alpha_{0}$ is a real number.

Almost all papers on forced excited Van der Pol systems deal with the potential $V_{2}$. However, some papers concerning the potential $V_{4}$ have shown a lot of interesting behaviours $[7,12,25,27,28]$. This case is usually referred to as Duffing-Van der Pol oscillator or $\phi^{4}$-Van der Pol oscillator. Nevertheless, more recently, some papers were published taking into account the potential $V_{6}$ meanly addressing the problem of chaos control $[13,17,22]$. The dynamics considering the potential $V_{6}$ is more complex and rich than the corresponding cases considering the potentials $V_{2}$ and $V_{4}$ [24,26]. This is the case that we will regard. This case is quoted in the literature as the extended Duffing-Van der Pol oscillator or $\phi^{6}$-Van der Pol oscillator.

In this paper, we will give an analytical treatment to system (1) in order to study its periodic solutions considering functions (2) and the potential $V_{6}$. Indeed, calling $y=\dot{x}$ we obtain

$$
\begin{aligned}
& \dot{x}=y, \\
& \dot{y}=-\omega_{0}^{2} x+\rho_{0} y-\alpha_{0} x^{3}-\rho_{0} x^{2} y-\lambda_{0} x^{5}+\delta_{0} \cos (\omega t) .
\end{aligned}
$$

System (3) becomes simpler if we perform a rescaling $s=\omega t$ in the time $t$ and another one $y=\omega_{0} Y$ in the spatial variable $y$. In fact, calling again the new time $s$ by $t$ and the variable $Y$ by $y$, after the rescaling, we have

$$
\begin{aligned}
& \dot{x}=y, \\
& \dot{y}=-x+\rho y-\alpha x^{3}-\rho x^{2} y-\lambda x^{5}+\delta \cos t,
\end{aligned}
$$

where the new parameters $\rho, \alpha, \lambda$ and $\delta$ are the respective old ones divided by $\omega_{0}^{2}$. The study of the periodic solutions of the non-linear non-autonomous $2 \pi$-periodic differential system (4) will be the objective of this paper.

When $\alpha, \lambda$ and $\delta$ are zero, Equation (4) is referred to as unforced Van der Pol equation and has a unique stable periodic solution for $\rho$ positive. Furthermore, if $\rho$ is large, this periodic solution remains, and it describes a periodic oscillatory behaviour called relaxation oscillation. On the other hand, by considering $\delta$ non-zero, system (4) can present non-linear attractors from simple periodic solutions up to multi-periodicity and chaos. In [10], we can find a didactical discussion about these objects and some results using classical techniques are obtained for a special case of system (4) using particular values of $\rho, \delta, \omega$ and initial conditions.

There exists an exhaustive list of papers in the literature studying the properties of system (4) when $\delta$ is zero. For $\delta$ positive, many open questions remain mainly due to the difficulty of integrating the system. In particular, in [19], Ma et al. study some aspects of robust practical synchronization for system (1) and apply the results to a particular case of system (4). In the same direction, in [14], Leung investigates synchronization processes between chaotic attractors 
considering $\alpha=\lambda=0$ in system (4). In [2,11], many aspects of system (4) for the case where $\alpha$ and $\lambda$ are zero and $\rho$ is large are studied. Furthermore, in [8], Egami and Hirano provide sufficient conditions to the existence of one periodic solution under some analytical hypotheses for a general forced Van der Pol system considering $\rho$ equal to zero.

Periodic solutions for system (4) were found in [17], where Liu and Yamaura investigate chaos control. Besides, in [5,6], results on the existence of periodic solutions for an autonomous special case of the extended Duffing-Van der Pol oscillator were obtained. In [16,30,31], we can also find some results on systems similar to system (4).

In this paper, we are concerned with periodic solutions of system (4). We will present sufficient conditions in order that this system possesses one or three periodic solutions, and we will provide conditions on the parameters $\rho, \alpha, \lambda$ and $\delta$ for which these bounds are realizable. In addition, we will prove that it is not possible to obtain different bounds of periodic solutions using the methodology presented in this paper.

The phase space of our non-autonomous differential system is $(x, y, t)$ and its Poincaré map is defined in the $(x, y)$-space. When possible the stability of the periodic solutions will be studied. As usual a periodic solution is stable if the eigenvalues of the fixed point associated with its Poincaré map have a negative real part; otherwise, the periodic solution is unstable. Inside the unstable periodic solutions there are two types: the unstable saddle periodic solutions having an eigenvalue with a negative real part and the other with a positive real part, and the repeller with both eigenvalues having a negative real part.

We should note that the method used here for studying periodic solutions can be applied to any periodic non-autonomous differential system as done in $[9,18]$. In these papers, the authors applied the method used in this paper in order to guarantee the existence of periodic solutions in a periodic FitzHugh-Nagumo system and in the Vallis system, respectively.

The paper is organized as follows. In Section 1.2, the main results are stated and compared with other results. Also, some important points on the results are clarified. In Section 2.1, we prove the results. In Section 2.2, some aspects of the results are pointed out. Lastly, Section 3 is devoted to give a brief summary of the results that we use from the averaging theory.

\subsection{Statement of the main results}

In this section, we present our results. We will behave under an analytical approach and for this reason the results are valid for a large range of the parameters of system (4), different from the major part of the results dealing with numerical techniques. In addition, it is important to note that we are concerned with harmonic solutions in the sense that they do not bifurcate from periodic solutions with multiple periods.

We have the following results.

THEOREM 1 Consider $\varepsilon>0$ sufficiently small, $(\rho, \delta, \alpha, \lambda)=\left(\varepsilon r, \varepsilon d, \varepsilon^{n_{2}} a, \varepsilon^{n_{3}} \ell\right)$ with $n_{2}, n_{3}>1$ and $81 d^{2}>48 r^{2}>0$. Then system (4) has a $2 \pi$-periodic solution $(x(t, \varepsilon), y(t, \varepsilon))$ such that

$$
(x(0, \varepsilon), y(0, \varepsilon)) \rightarrow\left(0, \frac{2(36 r)^{1 / 3}}{3 \Gamma}+\frac{1}{3}\left(\frac{6}{r}\right)^{1 / 3} \Gamma\right),
$$

when $\varepsilon \rightarrow 0$, where $\Gamma=\left(9 d+\sqrt{81 d^{2}-48 r^{2}}\right)^{1 / 3}$. Moreover, for $r$ sufficiently small, this periodic solution is stable. 
THeORem 2 Consider $\varepsilon>0$ sufficiently small, $(\alpha, \delta, \rho, \lambda)=\left(\varepsilon a, \varepsilon d, \varepsilon^{n_{1}} r, \varepsilon^{n_{3}} \ell\right)$ with $n_{1}, n_{3}>1$ and $\alpha \neq 0$. Then system (4) has a $2 \pi$-periodic solution $(x(t, \varepsilon), y(t, \varepsilon))$ such that

$$
(x(0, \varepsilon), y(0, \varepsilon)) \rightarrow\left(\left(\frac{4 d}{3 a}\right)^{1 / 3}, 0\right)
$$

when $\varepsilon \rightarrow 0$.

THEOREM 3 Consider $\varepsilon>0$ sufficiently small and $(\lambda, \delta, \rho, \alpha)=\left(\varepsilon \ell, \varepsilon d, \varepsilon^{n_{1}} r, \varepsilon^{n_{2}} a\right)$ with $n_{1}, n_{2}>1$. Then system (4) has a $2 \pi$-periodic solution $(x(t, \varepsilon), y(t, \varepsilon))$ such that

$$
(x(0, \varepsilon), y(0, \varepsilon)) \rightarrow\left(\left(\frac{8 d}{5 \ell}\right)^{1 / 5}, 0\right)
$$

when $\varepsilon \rightarrow 0$.

We remark that the stability of the periodic solutions of Theorems 2 and 3 cannot be decided with the real part of the eigenvalues of their Poincaré map because these real parts are zero.

THEOREM 4 Consider $\varepsilon>0$ sufficiently small and $(x, y, \rho, \delta, \alpha, \lambda)=\left(\varepsilon X, \varepsilon Y, \varepsilon r, \varepsilon^{2} d, \varepsilon^{n_{2}} a, \varepsilon^{n_{3}} \ell\right)$ with $\rho \neq 0$. Then system (4) has a $2 \pi$-periodic solution $(x(t, \varepsilon), y(t, \varepsilon))$ such that

$$
(x(0, \varepsilon), y(0, \varepsilon)) \rightarrow\left(O(\varepsilon),-\frac{\delta}{\varepsilon \rho}+O(\varepsilon)\right)
$$

when $\varepsilon \rightarrow 0$. Moreover, this periodic solution is unstable.

Note that in Theorem 4 we have a periodic solution that comes from infinity. As far as we know, this kind of behaviour also has not been observed in the papers concerned with system (4). Nevertheless, this behaviour is common when we perform a rescaling in the parameters and variables as we made previously and can be observed also in $[9,18]$.

THeOREM 5 Consider $\varepsilon>0$ sufficiently small, $(\alpha, \lambda, \delta, \rho)=\left(\varepsilon a, \varepsilon \ell, \varepsilon d, \varepsilon^{n_{1}} r\right)$ with $n_{1}>1$, $\alpha \lambda<0$ and

$$
D=\frac{53747712 a^{5} d^{2}}{78125 \ell^{7}}+\frac{20480 d^{4}}{\ell^{4}}
$$

Then system (4) has a $2 \pi$-periodic solution if $D=0$ and three $2 \pi$-periodic solutions if $D<0$. Moreover, there are values of $\alpha, \lambda$ and $\delta$ such that for all $\rho$ the system realizes the number of these periodic solutions.

Theorem 6 Consider $\varepsilon>0$ sufficiently small, $(\rho, \alpha, \delta, \lambda)=\left(\varepsilon r, \varepsilon a, \varepsilon d, \varepsilon^{n_{3}} \ell\right)$ with $n_{3}>1$ and $-324 a^{4} r^{2}-9 a^{2} d^{2} r^{2}+36 a^{2} r^{4}-d^{2} r^{4} \neq 0$. Consider also the values

$$
\Delta_{1}=324 a^{4}+d^{2} r^{2}+9 a^{2}\left(d^{2}-4 r^{2}\right)
$$




$$
\Delta_{2}=2187 a^{4} d^{4}+27 d^{4} r^{4}-16 d^{2} r^{6}+18 a^{2}\left(27 d^{4} r^{2}-72 d^{2} r^{4}+32 r^{6}\right) .
$$

The system

$$
\begin{aligned}
r x_{0}\left(-4+x_{0}^{2}+y_{0}^{2}\right)-3 a y_{0}\left(x_{0}^{2}+y_{0}^{2}\right) & =0, \\
4 d-r y_{0}\left(-4+x_{0}^{2}+y_{0}^{2}\right)-3 a x_{0}\left(x_{0}^{2}+y_{0}^{2}\right) & =0
\end{aligned}
$$

has one solution $\left(x_{0}^{0}, y_{0}^{0}\right)$ if $\Delta_{1} \Delta_{2}=0$ or $\Delta_{2}>0$, and three solutions $\left(x_{0}^{i}, y_{0}^{i}\right)$ if $\Delta_{2}<0$ for $i=$ $1,2,3$; we assume that all of them satisfy

$$
27 \alpha^{2}\left(\left(x_{0}^{i}\right)^{2}+\left(y_{0}^{i}\right)^{2}\right)^{2}+\rho^{2}\left(-4+\left(x_{0}^{i}\right)^{2}+\left(y_{0}^{i}\right)^{2}\right)\left(-4+3\left(x_{0}^{i}\right)^{2}+3\left(y_{0}^{i}\right)^{2}\right) \neq 0 .
$$

Then if $\Delta_{1} \Delta_{2}=0$ or $\Delta_{2}>0$, system (4) has a $2 \pi$-periodic solution $\left(x^{0}(t, \varepsilon), y^{0}(t, \varepsilon)\right)$ such that $\left(x^{0}(0, \varepsilon), y^{0}(0, \varepsilon)\right) \rightarrow\left(x_{0}^{0}, y_{0}^{0}\right)$ when $\varepsilon \rightarrow 0$. Additionally if $\Delta_{2}<0$ system (4) has three $2 \pi$-periodic solutions $\left(x^{i}(t, \varepsilon), y^{i}(t, \varepsilon)\right)$ such that $\left(x^{i}(0, \varepsilon), y^{i}(0, \varepsilon)\right) \rightarrow\left(x_{0}^{i}, y_{0}^{i}\right)$ when $\varepsilon \rightarrow 0$ for $i=1,2,3$. Moreover, there are values of $\rho, \alpha$ and $\delta$ such that for all $\lambda$ the system realizes the number of these periodic solutions.

TheOREM 7 Consider $\varepsilon>0$ sufficiently small, $(\alpha, \rho, \lambda, \delta)=(\varepsilon a, \varepsilon r, \varepsilon \ell, \varepsilon d), \quad \alpha \rho \neq 0$, $\left(r^{2}-3 a^{2}\right)\left(r^{2}-9 a^{2}\right) \neq 0$ and

$$
\begin{aligned}
C= & 4(3 a+10 \ell)\left(540 a^{3} \ell-9 a^{2}\left(d^{2}-600 \ell^{2}\right)-120 a \ell\left(d^{2}-150 \ell^{2}\right)\right. \\
& \left.+50 \ell^{2}\left(-7 d^{2}+400 \ell^{2}\right)\right) r^{6}+6(a+5 \ell)(6 a-d+20 \ell)(6 a+d+20 \ell) r^{8} \neq 0, \\
D= & 2066242608 a^{14} d^{6}-3125 d^{8} r^{12}-531441 a^{12}\left(3125 d^{8}+96 d^{6} r^{2}+1536 d^{4} r^{4}-1024 d^{2} r^{6}\right) \\
& +354294 a^{10}\left(3125 d^{8} r^{2}+616 d^{6} r^{4}-1600 d^{4} r^{6}\right) \\
& +18 a^{2} r^{10}\left(9375 d^{8}+5000 d^{6} r^{2}-88000 d^{4} r^{4}+102400 d^{2} r^{6}-32768 r^{8}\right) \\
& +2916 a^{6} r^{6}\left(15625 d^{8}+11700 d^{6} r^{2}-45632 d^{4} r^{4}+23552 d^{2} r^{6}-2048 r^{8}\right) \\
& -6561 a^{8} r^{4}\left(46875 d^{8}+24832 d^{6} r^{2}-66816 d^{4} r^{4}+12288 d^{2} r^{6}+4096 r^{8}\right) \\
& +81 a^{4} r^{8}\left(-46875 d^{8}-36000 d^{6} r^{2}+275200 d^{4} r^{4}-246784 d^{2} r^{6}+61440 r^{8}\right) .
\end{aligned}
$$

The system

$$
\begin{aligned}
& 2 r x_{0}\left(-4+x_{0}^{2}+y_{0}^{2}\right)-y_{0}\left(x_{0}^{2}+y_{0}^{2}\right)\left(6 a+5 \ell\left(x_{0}^{2}+y_{0}^{2}\right)\right)=0 \\
& 8 d+8 r y_{0}-\left(x_{0}^{2}+y_{0}^{2}\right)\left(6 a x_{0}+2 r y_{0}+5 \ell x_{0}\left(x_{0}^{2}+y_{0}^{2}\right)\right)=0
\end{aligned}
$$

has one solution $\left(x_{0}^{0}, y_{0}^{0}\right)$ if $D<0$, and three solutions $\left(x_{0}^{i}, y_{0}^{i}\right)$ if $D>0$ for $i=1,2,3$; we assume that all of them satisfy

$$
\begin{aligned}
& 4 \rho^{2}\left(-4+\left(x_{0}^{i}\right)^{2}+\left(y_{0}^{i}\right)^{2}\right)\left(-4+3\left(x_{0}^{i}\right)^{2}+3\left(y_{0}^{i}\right)^{2}\right) \\
& \quad+\left(\left(x_{0}^{i}\right)^{2}+\left(y_{0}^{i}\right)^{2}\right)^{2}\left(6 \alpha+\rho \lambda\left(\left(x_{0}^{i}\right)^{2}+\left(y_{0}^{i}\right)^{2}\right)\right)\left(18 \alpha+25 \lambda\left(x_{0}^{i}\right)^{2}+3\left(y_{0}^{i}\right)^{2}\right) \neq 0 .
\end{aligned}
$$

Then, if $D<0$ system (4) has a $2 \pi$-periodic solution $\left(x^{0}(t, \varepsilon), y^{0}(t, \varepsilon)\right)$ such that $\left(x^{0}(0, \varepsilon), y^{0}(0, \varepsilon)\right) \rightarrow\left(x_{0}^{0}, y_{0}^{0}\right)$ when $\varepsilon \rightarrow 0$. Furthermore, if $D>0$ system (4) has three $2 \pi$ periodic solutions $\left(x^{i}(t, \varepsilon), y^{i}(t, \varepsilon)\right)$ such that $\left(x^{i}(0, \varepsilon), y^{i}(0, \varepsilon)\right) \rightarrow\left(x_{0}^{i}, y_{0}^{i}\right)$ when $\varepsilon \rightarrow 0$ for $i=$ $1,2,3$. Moreover, there are values of $\alpha, \rho, \lambda$ and $\delta$ for which the system realizes the number of these periodic solutions. 
THeOREM 8 Consider $\varepsilon>0$ sufficiently small, $(\rho, \lambda, \delta, \alpha)=\left(\varepsilon r, \varepsilon \ell, \varepsilon d, \varepsilon^{n_{2}} a\right)$ with $n_{2}>1$. Consider the numbers

$$
\begin{aligned}
C= & 4000 d^{3} \ell^{3} r^{4}+7200000 d \ell^{5} r^{4}+60 d^{3} \ell r^{6}+72000 d \ell^{3} r^{6}+\left(50 d^{4} \ell^{2} r^{4}-220000 d^{2} \ell^{4} r^{4}\right. \\
& \left.-8000000 \ell^{6} r^{4}-2800 d^{2} \ell^{2} r^{6}+160000 \ell^{4} r^{6}-6 d^{2} r^{8}+2400 \ell^{2} r^{8}\right) \\
N_{2}= & -4500 \ell^{2} r^{4}+3 r^{6} \\
N_{3}= & 5859375 d^{2} \ell^{4} \ell^{6}+18750000 d^{2} \ell^{6} r^{2}+87500 \ell^{4}\left(-7 d^{2}+1200 \ell^{2}\right) r^{4} \\
& +25 \ell^{2}\left(-7 d^{2}+155600 \ell^{2}\right) r^{6}+15600 \ell^{2} r^{8}+9 r^{10}, \\
N_{4}= & 1171875 d^{8} \ell^{6}\left(1250000 \ell^{4}+8500 \ell^{2} r^{2}+r^{4}\right)-2500 d^{6} \ell^{4}\left(312500000000 \ell^{8}\right. \\
& \left.+5343750000 \ell^{6} r^{2}+74625000 \ell^{4} r^{4}+358375 \ell^{2} r^{6}+69 r^{8}\right) \\
& -3000 d^{4} \ell^{2} r^{2}\left(218750000000 \ell^{10}-21625000000 \ell^{8} r^{2}-686625000 \ell^{6} r^{4}\right. \\
& \left.-3715000 \ell^{4} r^{6}-650 \ell^{2} r^{8}-3 r^{10}\right)-1600 \ell^{2} r^{6}\left(400000000000 \ell^{10}\right. \\
& -144900000000 \ell^{8} r^{2}-2817000000 \ell^{6} r^{4}-8880000 \ell^{4} r^{6}+38700 \ell^{2} r^{8} \\
& \left.+27 r^{10}\right)+12 d^{2} r^{4}\left(-50000000000000 \ell^{12}-32250000000000 \ell^{10} r^{2}\right. \\
& \left.-662400000000 \ell^{8} r^{4}-2803000000 \ell^{6} r^{6}+5410000 \ell^{4} r^{8}+19200 \ell^{2} r^{10}+9 r^{12}\right) \\
N_{5}= & 48828125 d^{8} \ell^{6}-4687500 d^{6} \ell^{4} r^{4}+6400 \ell^{2} r^{10}\left(1600 \ell^{2}+r^{2}\right)-16 d^{2} r^{8} \\
& \left(2000000 \ell^{4}+2900 \ell^{2} r^{2}+r^{4}\right)+d^{4} r^{6}\left(27500000 \ell^{4}+60000 \ell^{2} r^{2}+27 r^{4}\right) \\
M_{5}= & -25 d^{4} \ell 2+d^{2}\left(110000 \ell 4+1400 \ell 2 r^{2}+3 r^{4}\right)+400\left(10000 \ell 6-200 \ell^{4} r^{2}-3 \ell^{2} r^{4}\right)
\end{aligned}
$$

We assume that $C M_{5} \neq 0$. The system

$$
\begin{aligned}
2 r x_{0}\left(-4+x_{0}^{2}+y_{0}^{2}\right)-5 \ell y_{0}\left(x_{0}^{2}+y_{0}^{2}\right)^{2} & =0, \\
8 d-2 r y_{0}\left(-4+x_{0}^{2}+y_{0}^{2}\right)-5 \ell x_{0}\left(x_{0}^{2}+y_{0}^{2}\right)^{2} & =0
\end{aligned}
$$

has one solution $\left(x_{0}^{0}, y_{0}^{0}\right)$ if $N_{2} \leq 0, N_{3} \geq 0$ or $N_{4} \geq 0$ and $N_{5}>0$, and three solutions $\left(x_{0}^{i}, y_{0}^{i}\right)$ if $N_{5}<0$ for $i=1,2,3$; we assume that all of them satisfy

$$
125 \lambda^{2}\left(\left(x_{0}^{i}\right)^{2}+\left(y_{0}^{i}\right)^{4}\right)+4 \rho^{2}\left(-4+\left(x_{0}^{i}\right)^{2}+\left(y_{0}^{i}\right)^{2}\right)\left(-4+3\left(x_{0}^{i}\right)^{2}+3\left(y_{0}^{i}\right)^{2}\right) \neq 0 .
$$

Hence, if $N_{2} \leq 0, N_{3} \geq 0$ or $N_{4} \geq 0$ and $N_{5}>0$, then system (4) has a $2 \pi$-periodic solution $\left(x^{0}(t, \varepsilon), y^{0}(t, \varepsilon)\right)$ such that $\left(x^{0}(0, \varepsilon), y^{0}(0, \varepsilon)\right) \rightarrow\left(x_{0}^{0}, y_{0}^{0}\right)$ when $\varepsilon \rightarrow 0$. If $N_{5}<0$ then system (4) has three $2 \pi$-periodic solutions $\left(x^{i}(t, \varepsilon), y^{i}(t, \varepsilon)\right)$ such that $\left(x^{i}(0, \varepsilon), y^{i}(0, \varepsilon)\right) \rightarrow\left(x_{0}^{i}, y_{0}^{i}\right)$ when $\varepsilon \rightarrow 0$ for $i=1,2,3$. Moreover, there are values of $\rho, \lambda$ and $\delta$ such that for all $\alpha$ system (4) has one or three periodic solutions.

We note that in Theorems 5, 6, 7 and 8 we do not say anything about the kind of stability of the periodic solutions because we do not have the explicit expressions of the real part of the eigenvalues of their Poincaré maps.

We remark that the periodic solutions provided in Theorems 1 until 8 exist when we take small values for the parameters of system (4) obeying some relations among them. For instance, Theorem 1 states the existence of one periodic solution for system (4) if each parameter of this system is small and $\alpha$ and $\lambda$ are much smaller than $\rho$ and $\delta$. This assertion becomes more clear 
if we observe the replacement done in each theorem and take into account that $\varepsilon$ is sufficiently small. As far as we know, periodic solutions of system (4) whose parameters have this characteristic have not been observed in the literature. Besides, it seems that the simultaneous bifurcation of three harmonic periodic solutions in the extended Duffing-Van der Pol system is also new.

Furthermore, we note that the periodic solutions presented in the present paper are different from those stated in [8]. Indeed, in the referred paper, the authors ask for $V_{6}^{\prime}(0)<0$, and in our case, we have $V_{6}^{\prime}(0)=0$.

\section{Proof and discussion of the results}

\subsection{Proof of the results}

In order to apply the averaging theory described in Section 3 in systems (4), we start performing a rescaling of the variables $x$ and $y$ and of the parameters $\rho, \alpha, \lambda$ and $\delta$ as follows:

$$
\begin{aligned}
& x=\varepsilon^{m_{1}} X, \quad y=\varepsilon^{m_{2}} Y, \\
& \rho=\varepsilon^{n_{1}} r, \quad \alpha=\varepsilon^{n_{2}} a, \quad \lambda=\varepsilon^{n_{3}} \ell, \quad \delta=\varepsilon^{n_{4}} d,
\end{aligned}
$$

where $\varepsilon$ is positive and sufficiently small and $m_{i}$ and $n_{j}$ are non-negative integers, for $i=1,2$ and $j=1,2,3,4$. We recall that since $\delta>0, \rho \geq 0$ and $\lambda \neq 0$ we have $d>0, r \geq 0$ and $\ell \neq 0$.

In the new variables $(X, Y)$, system (4) is written as

$$
\begin{aligned}
& \frac{\mathrm{d} X}{\mathrm{~d} t}=\varepsilon^{-m_{1}+m_{2}} Y, \\
& \frac{\mathrm{d} Y}{\mathrm{~d} t}=-\varepsilon^{m_{1}-m_{2}} X+\varepsilon^{n_{1}} r Y-\varepsilon^{2 m_{1}+n_{1}} r X^{2} Y-\varepsilon^{3 m_{1}-m_{2}+n_{2}} a X^{3}-\varepsilon^{5 m_{1}-m_{2}+n_{3}} \ell X^{5}+\varepsilon^{-m_{2}+n_{4}} d \cos t .
\end{aligned}
$$

In order to have non-negative powers of $\varepsilon$, we must impose the conditions

$$
m_{1}=m_{2}=m \quad \text { and } \quad n_{4} \geq m,
$$

where $m$ is a non-negative integer.

Hence, with conditions (7), system (6) becomes

$$
\begin{aligned}
& \frac{\mathrm{d} X}{\mathrm{~d} t}=Y, \\
& \frac{\mathrm{d} Y}{\mathrm{~d} t}=-X+\varepsilon^{n_{1}} r Y-\varepsilon^{2 m+n_{1}} r X^{2} Y-\varepsilon^{2 m+n_{2}} a X^{3}-\varepsilon^{4 m+n_{3}} \ell X^{5}+\varepsilon^{-m+n_{4}} d \cos t
\end{aligned}
$$

In this paper, we will find periodic solutions of system (8) depending on the parameters $r, a, \ell$ and $d$ and the powers $m, n_{i}$ of $\varepsilon$, for $i=1,2,3,4$. Then, we will go back through rescaling (5) to ensure the existence of periodic solutions in system (4).

From now on we assume that the values $n_{1}, m^{2}+n_{2}^{2}, m^{2}+n_{3}^{2}$ and $n_{4}-m$ are positive and observe that considering these conditions each power of $\varepsilon$ in system (8) becomes positive. The reason for a such assumption will be explained later on in Section 2.2. Now we shall apply the averaging theory described in Section 3. Thus following the notation of the mentioned section and denoting again the variables $(X, Y)$ by $(x, y)$, we have $\mathbf{x}=(x, y)^{\mathrm{T}}$, and system (13) 
corresponding to system (8) can be written as

$$
\dot{\mathbf{x}}=F_{0}(t, \mathbf{x})=(y,-x)^{\mathrm{T}} .
$$

So the solution $\mathbf{x}(t, \mathbf{z})=(x(t, \mathbf{z}), y(t, \mathbf{z}))$ of system (9) such that $\mathbf{x}(0, \mathbf{z})=\mathbf{z}=\left(x_{0}, y_{0}\right)$ is

$$
\begin{aligned}
& x(t, \mathbf{z})=x_{0} \cos t+y_{0} \sin t, \\
& y(t, \mathbf{z})=y_{0} \cos t-x_{0} \sin t .
\end{aligned}
$$

It is clear that the origin of coordinates of $\mathbb{R}^{2}$ is a global isochronous centre for system (9) whose circular periodic solutions starting on $\mathbf{z}=\left(x_{0}, y_{0}\right) \in \mathbb{R}^{2}$ are parametrized by the above functions $x(t, \mathbf{z})$ and $y(t, \mathbf{z})$. Therefore, through every initial condition $\left(x_{0}, y_{0}\right)$ in $\mathbb{R}^{2}$ passes a $2 \pi$-periodic solution of system (9).

Physically speaking, system (9) models a simple harmonic oscillator and its solutions $(x(t, \mathbf{z}), y(t, \mathbf{z}))$ describe the wave behaviour of this oscillator. In this direction, the problem of perturbation of the global centre (9) is equivalent to the problem of perturbation of a simple harmonic oscillator introducing a damping parameter as external force and considering a potential $V$ by taking small values for $\rho, \alpha, \lambda$ and $\delta$ as stated in Theorems $1-8$.

We note that using the notation of Section 3, the fundamental matrix $Y(t, \mathbf{z})$ of system (9) satisfying that $Y(0, \mathbf{z})$ is the identity of $\mathbb{R}^{2}$ is written as

$$
Y(t, \mathbf{z})=\left(\begin{array}{cc}
\cos t & \sin t \\
-\sin t & \cos t
\end{array}\right) .
$$

From the averaging theory we are interested in the simple zeros of the function

$$
f(\mathbf{z})=\left(f_{1}(\mathbf{z}), f_{2}(\mathbf{z})\right)=\int_{0}^{2 \pi} Y^{-1}(t, \mathbf{z}) F_{1}(t, \mathbf{x}(t, \mathbf{z}, 0)) \mathrm{d} t,
$$

where $F_{1}(t, \mathbf{x})$ are the terms of order 1 on $\varepsilon$ of the vector field associated with system (8)

$$
\left(y,-x+\varepsilon^{n_{1}} r y-\varepsilon^{2 m+n_{1}} r x^{2} y-\varepsilon^{2 m+n_{2}} a x^{3}-\varepsilon^{4 m+n_{3}} \ell x^{5}+\varepsilon^{-m+n_{4}} d \cos t\right)^{\mathrm{T}} .
$$

Now we prove our results.

Proof of Theorem 1 First we take $n_{1}=n_{4}=1, m=0$ and consider $n_{2}, n_{3}>1$. So rescaling (5) becomes $(x, y, \rho, \delta, \alpha, \lambda)=\left(X, Y, \varepsilon r, \varepsilon d, \varepsilon^{n_{2}} a, \varepsilon^{n_{3}} d\right)$ and calling again $(X, Y)$ by $(x, y)$ we verify the hypotheses of Theorem 1. Now the vector field of system (4) becomes

$$
\left(y,-x+\varepsilon r y-\varepsilon r x^{2} y-\varepsilon^{n_{2}} a x^{3}-\varepsilon^{n_{3}} \ell x^{5}+\varepsilon d \cos t\right)^{\mathrm{T}} .
$$

Hence, as we stated before, the terms of order 1 in this vector field are given by $F_{1}(t, \mathbf{x})=$ $\left(0, r y-r x^{2} y+d \cos t\right)$. Then since $\mathbf{z}=\left(x_{0}, y_{0}\right)$, the function $f\left(x_{0}, y_{0}\right)=\left(f_{1}\left(x_{0}, y_{0}\right), f_{2}\left(x_{0}, y_{0}\right)\right)$ given in Equation (10) turns into the form

$$
\begin{aligned}
f_{1}\left(x_{0}, y_{0}\right)= & \int_{0}^{2 \pi}-(\sin t)\left(d \cos t+r\left(y_{0} \cos t-x_{0} \sin t\right)\right. \\
& \left.-r\left(y_{0} \cos t-x_{0} \sin t\right)\left(x_{0} \cos t+y_{0} \sin t\right)^{2}\right) \mathrm{d} t
\end{aligned}
$$




$$
\begin{aligned}
= & -\frac{1}{4} \pi r x_{0}\left(-4+x_{0}^{2}+y_{0}^{2}\right) \\
f_{2}\left(x_{0}, y_{0}\right)= & \int_{0}^{2 \pi}(\cos t)\left(d \cos t+r\left(y_{0} \cos t-x_{0} \sin t\right)\right. \\
& \left.-r\left(y_{0} \cos t-x_{0} \sin t\right)\left(x_{0} \cos t+y_{0} \sin t\right)^{2}\right) \mathrm{d} t \\
= & \frac{1}{4}\left(4 d-r y_{0}\left(-4+x_{0}^{2}+y_{0}^{2}\right)\right) .
\end{aligned}
$$

The zeros of functions $f_{1}$ and $f_{2}$ are a pair of conjugate complex vectors and a real pair $\left(x_{0}^{0}, y_{0}^{0}\right)$ satisfying $x_{0}^{0}=0$ and

$$
y_{0}^{0}=\frac{2}{3} \frac{(36 r)^{1 / 3}}{\left(9 d+\sqrt{81 d^{2}-48 r^{2}}\right)^{1 / 3}}+\frac{1}{3}\left(\frac{6}{r}\right)^{1 / 3}\left(9 d+\sqrt{81 d^{2}-48 r^{2}}\right)^{1 / 3} .
$$

Note that $y_{0}^{0}$ is real because $81 d^{2}>48 r^{2}>0$ by assumption. We observe that the expression of $y_{0}^{0}=y_{0}^{0}(r, d)$ does not change when we go back through rescaling (5) taking $r=\varepsilon^{-1} \rho$ and $d=\varepsilon^{-1} \delta$ according to the hypotheses of Theorem 1 .

Moreover, we denote $\Gamma=\left(9 d+\sqrt{81 d^{2}-48 r^{2}}\right)^{1 / 3}$ and observe that the matrix $M=\left(m_{i j}\right)=$ $\partial\left(f_{1}, f_{2}\right) / \partial\left(x_{0}, y_{0}\right)$ at $\left(x_{0}^{0}, y_{0}^{0}\right)$ is diagonal and its elements are $m_{11}=-\pi r \Gamma_{+} / 36$ and $m_{22}=\pi r \Gamma_{-}$, where

$$
\Gamma_{ \pm}=-12 \pm \frac{6^{1 / 3} 24 r^{2 / 3}}{\Gamma^{2}} \pm \frac{6^{2 / 3} \Gamma^{2}}{r^{2 / 3}} .
$$

The determinant $\Pi_{0}$ of $M$ written in a power series of $r$ around $r=0$ is

$$
\Pi_{0}=\frac{3}{8}\left(2 \pi^{2} d(4 d)^{1 / 3}\right) r^{2 / 3}+\frac{(\pi r)^{2}}{3}+O\left(r^{7 / 3}\right),
$$

which is positive for $|r|$ sufficiently small because $d>0$. So the averaging theory described in Section 3 guarantees the existence of a $2 \pi$-periodic solution $(x(t, \varepsilon), y(t, \varepsilon))$ such that $(x(0, \varepsilon), y(0, \varepsilon))$ tends to $\left(x_{0}^{0}, y_{0}^{0}\right)$ when $\varepsilon \rightarrow 0$.

On the other hand, the trace $\Sigma_{0}$ of $M$ at $\left(x_{0}^{0}, y_{0}^{0}\right)$ is

$$
\Sigma_{0}=-2^{2 / 3} \pi(2 d)^{2 / 3} r^{1 / 3}-\frac{2 \pi r}{3}+O\left(r^{4 / 3}\right)
$$

Thus for $|r|$ sufficiently small, the trace $\Sigma_{0}$ of $M$ is negative. So the real part of the eigenvalues of the matrix $M$ are both negative and then the periodic solution $(x(t, \varepsilon), y(t, \varepsilon))$ is stable for each $\varepsilon$ sufficiently small. So Theorem 1 is proved.

Proof of Theorem 2 Now we take $n_{2}=n_{4}=1$ and consider $n_{1}, n_{3}>1$. So we are under the hypotheses of Theorem 2. Now, taking $m=0$ and doing rescaling (5), the vector field of system (8) is written as

$$
\left(y,-x+\varepsilon^{n_{1}} r y-\varepsilon^{n_{1}} r x^{2} y-\varepsilon a x^{3}-\varepsilon^{n_{3}} \ell x^{5}+\varepsilon d \cos t\right)^{\mathrm{T}} .
$$


Thus, we have $F_{1}(t, \mathbf{x})=\left(0,-a x^{3}+d \cos t\right)$, and $f\left(x_{0}, y_{0}\right)=\left(f_{1}\left(x_{0}, y_{0}\right), f_{2}\left(x_{0}, y_{0}\right)\right)$ becomes

$$
\begin{aligned}
f_{1}\left(x_{0}, y_{0}\right) & =\int_{0}^{2 \pi}-(\sin t)\left(d \cos t-a\left(x_{0} \cos t+y_{0} \sin t\right)^{3}\right) \mathrm{d} t \\
& =\frac{3}{4} a \pi y_{0}\left(x_{0}^{2}+y_{0}^{2}\right), \\
f_{2}\left(x_{0}, y_{0}\right) & =\int_{0}^{2 \pi}(\cos t)\left(d \cos t-a\left(x_{0} \cos t+y_{0} \sin t\right)^{3}\right) \mathrm{d} t \\
& =\frac{1}{4} \pi\left(4 d-3 a x_{0}\left(x_{0}^{2}+y_{0}^{2}\right)\right) .
\end{aligned}
$$

The real zero $\left(x_{0}^{0}, y_{0}^{0}\right)$ of $f_{1}$ and $f_{2}$ is

$$
\left(x_{0}^{0}, y_{0}^{0}\right)=\left(\left(\frac{4 d}{3 a}\right)^{1 / 3}, 0\right) .
$$

Again the values $\left(x_{0}^{0}, y_{0}^{0}\right)$ does not depend on $\varepsilon$ when we go back to the original parameters $\alpha$ and $\delta$ through rescaling (5). Besides, the matrix $M=\partial\left(f_{1}, f_{2}\right) / \partial\left(x_{0}, y_{0}\right)$ at $\left(x_{0}^{0}, y_{0}^{0}\right)$ now is written as

$$
M=\frac{1}{\varepsilon}\left(\begin{array}{cc}
0 & \left(\left(\frac{3}{4}\right) \alpha \delta^{2}\right)^{1 / 3} \pi \\
-3\left(\left(\frac{3}{4}\right) \alpha \delta^{2}\right)^{1 / 3} \pi & 0
\end{array}\right),
$$

and then the determinant $\Pi_{0}$ of $M$ is

$$
\Pi_{0}=\frac{3 \pi^{2}}{2 \varepsilon^{2}}\left(\frac{9 \alpha^{2} \delta^{4}}{2}\right)^{1 / 3}>0 .
$$

Therefore, the averaging theory implies the existence of a $2 \pi$-periodic solution $(x(t, \varepsilon), y(t, \varepsilon))$ such that $(x(0, \varepsilon), y(0, \varepsilon))$ tends to $\left(x_{0}^{0}, y_{0}^{0}\right)$ when $\varepsilon \rightarrow 0$.

We observe that since the trace $\Sigma_{0}$ of $M$ is zero and the determinant $\Pi_{0}$ is positive, then the real part of both eigenvalues of $M$ is zero. So we cannot decide about the stability of the periodic solution of Theorem 2. In what follows, we see that the same occurs in Theorem 3.

Proof of Theorem 3 We start considering $n_{3}=n_{4}=1, n_{1}, n_{2}>1$ and $m=0$. Therefore, we are under the assumptions of Theorem 3. Doing rescaling (5) the vector field of system (8) becomes

$$
\left(y,-x+\varepsilon^{n_{1}} r y-\varepsilon^{n_{1}} r x^{2} y-\varepsilon^{n_{2}} a x^{3}-\varepsilon \ell x^{5}+\varepsilon d \cos t\right)^{\mathrm{T}} .
$$

So we conclude that $F_{1}(t, \mathbf{x})=\left(0,-\ell x^{5}+d \cos t\right)$ and then we get

$$
\begin{aligned}
f_{1}\left(x_{0}, y_{0}\right) & =\int_{0}^{2 \pi}-(\sin t)\left(d \cos t-\ell\left(x_{0} \cos t+y_{0} \sin t\right)^{5}\right) \mathrm{d} t \\
& =\frac{5}{8} \ell \pi y_{0}\left(x_{0}^{2}+y_{0}^{2}\right)^{2}
\end{aligned}
$$




$$
\begin{aligned}
f_{2}\left(x_{0}, y_{0}\right) & =\int_{0}^{2 \pi}(\cos t)\left(d \cos t-\ell\left(x_{0} \cos t+y_{0} \sin t\right)^{5}\right) \mathrm{d} t \\
& =\frac{1}{8} \pi\left(8 d-5 \ell x_{0}\left(x_{0}^{2}+y_{0}^{2}\right)^{2}\right) .
\end{aligned}
$$

Now the real zero $\left(x_{0}^{0}, y_{0}^{0}\right)$ of $f_{1}$ and $f_{2}$ is

$$
\left(x_{0}^{0}, y_{0}^{0}\right)=\left(\left(\frac{8 d}{5 \ell}\right)^{1 / 5}, 0\right) .
$$

We note that as in Theorems 1 and 2 the root $\left(x_{0}^{0}, y_{0}^{0}\right)$ does not depend on $\varepsilon$ after we perform rescaling (5). However, using Equation (5), the matrix $M=\partial\left(f_{1}, f_{2}\right) / \partial\left(x_{0}, y_{0}\right)$ at $\left(x_{0}^{0}, y_{0}^{0}\right)$ is

$$
M=\frac{1}{\varepsilon}\left(\begin{array}{cc}
0 & \left(\left(\frac{5}{8}\right) \lambda \delta^{4}\right)^{1 / 5} \pi \\
-5\left(\left(\frac{5}{8}\right) \lambda \delta^{4}\right)^{1 / 5} \pi & 0
\end{array}\right),
$$

whose determinant is

$$
\Pi_{0}=\frac{5 \pi^{2}}{2 \varepsilon^{2}}\left(\frac{25 \lambda^{2} \delta^{8}}{2}\right)^{1 / 5}>0 .
$$

Then the averaging method states the existence of a $2 \pi$-periodic solution $(x(t, \varepsilon), y(t, \varepsilon))$ such that $(x(0, \varepsilon), y(0, \varepsilon))$ tends to $\left(x_{0}^{0}, y_{0}^{0}\right)$ when $\varepsilon \rightarrow 0$. The stability of this periodic solution is unknown because the trace $\Sigma_{0}$ of $M$ is zero and the determinant $\Pi_{0}>0$ is positive just as in Theorem 2.

Proof of Theorem 4 In what follows, we assume that $n_{1}=m=1$ and $n_{4}=2$. Now the expression of the vector field of system (8) is

$$
\left(y,-x+\varepsilon r y-\varepsilon^{3} r x^{2} y-\varepsilon^{n_{2}+2} a x^{3}-\varepsilon^{n_{3}+4} \ell x^{5}+\varepsilon d \cos t\right)^{\mathrm{T}},
$$

and then $F_{1}(t, \mathbf{x})=(0, r y+d \cos t)$. In this case, we obtain

$$
\begin{aligned}
f_{1}\left(x_{0}, y_{0}\right) & =\int_{0}^{2 \pi}-(\sin t)\left(d \cos t+r\left(y_{0} \cos t-x_{0} \sin t\right)\right) \mathrm{d} t \\
& =\pi r x_{0}, \\
f_{2}\left(x_{0}, y_{0}\right) & =\int_{0}^{2 \pi}(\cos t)\left(d \cos t+r\left(y_{0} \cos t-x_{0} \sin t\right)\right) \mathrm{d} t \\
& =\pi\left(d+r y_{0}\right) .
\end{aligned}
$$

It is immediate that the only zero $\left(x_{0}^{0}, y_{0}^{0}\right)$ of $f_{1}$ and $f_{2}$ in this case is $\left(x_{0}^{0}, y_{0}^{0}\right)=(0, d / r)$. So after going back through rescaling (5) taking $r=\varepsilon^{-1} \rho$ and $d=\varepsilon^{-2} \delta$, we have

$$
\left(x_{0}^{0}, y_{0}^{0}\right)=\left(0,-\frac{\delta}{\varepsilon \rho}\right) \text {. }
$$

In addition, the matrix $M=\partial\left(f_{1}, f_{2}\right) / \partial\left(x_{0}, y_{0}\right)$ at $\left(x_{0}^{0}, y_{0}^{0}\right)$ is diagonal and its elements are both given by $\rho \pi / \varepsilon$. Consequently, $M$ has determinant $\Pi_{0}=(\rho \pi / \varepsilon)^{2}>0$ and trace $\Sigma_{0}=2 \rho \pi / \varepsilon>$ 
0 . Then the averaging theory described in Section 3 assures the existence of a $2 \pi$-periodic solution $(x(t, \varepsilon), y(t, \varepsilon))$ such that $(x(0, \varepsilon), y(0, \varepsilon))$ tends to $\left(x_{0}^{0}, y_{0}^{0}\right)$ when $\varepsilon \rightarrow 0$. Moreover, since $\rho$ is positive this periodic solution is unstable. Then, Theorem 4 is proved.

Proof of Theorem 5 Now we take $m=0, n_{2}=n_{3}=n_{4}=1$ and $n_{1}>1$. The expression of the vector field of system (8) in this case is

$$
\left(y,-x+\varepsilon^{n_{1}} r y-\varepsilon^{n_{1}} r x^{2} y-\varepsilon a x^{3}-\varepsilon \ell x^{5}+\varepsilon d \cos t\right)^{\mathrm{T}},
$$

and we get $F_{1}(t, \mathbf{x})=\left(0,-a x^{3}-\ell x^{5}+d \cos t\right)$. Hence, function $f=\left(f_{1}, f_{2}\right)$ is written as

$$
\begin{aligned}
f_{1}\left(x_{0}, y_{0}\right) & =\int_{0}^{2 \pi}-(\sin t)\left(d \cos t-a\left(x_{0} \cos t+y_{0} \sin t\right)^{3}-\ell\left(x_{0} \cos t+y_{0} \sin t\right)^{5}\right) \mathrm{d} t \\
& =\frac{1}{8} \pi y_{0}\left(x_{0}^{2}+y_{0}^{2}\right)\left(6 a+5 \ell\left(x_{0}^{2}+y_{0}^{2}\right)\right), \\
f_{2}\left(x_{0}, y_{0}\right) & =\int_{0}^{2 \pi}(\cos t)\left(d \cos t-a\left(x_{0} \cos t+y_{0} \sin t\right)^{3}-\ell\left(x_{0} \cos t+y_{0} \sin t\right)^{5}\right) \mathrm{d} t \\
& =\frac{1}{8} \pi\left(8 d-x_{0}\left(x_{0}^{2}+y_{0}^{2}\right)\left(6 a+5 \ell\left(x_{0}^{2}+y_{0}^{2}\right)\right)\right) .
\end{aligned}
$$

In order to find the zeros of $f\left(x_{0}, y_{0}\right)$, we compute a Gröbner basis $\left\{b_{k}\left(x_{0}, y_{0}\right), k=1,2,3\right\}$ in the variables $x_{0}$ and $y_{0}$ for the set of polynomials $\left\{\bar{f}_{1}\left(x_{0}, y_{0}\right), \bar{f}_{2}\left(x_{0}, y_{0}\right)\right\}$, where $\bar{f}_{1,2}=(8 / \pi) f_{1,2}$. Then, we look for the zeros of each $b_{k}, k=1,2,3$. It is a known fact that the zeros of a Gröbner basis of $\left.\left\{\bar{f}_{1}\left(x_{0}, y_{0}\right)\right), \bar{f}_{2}\left(x_{0}, y_{0}\right)\right\}$ are the zeros of $\bar{f}_{1}$ and $\bar{f}_{2}$, and consequently, zeros of $f_{1}$ and $f_{2}$ too. For more information about a Gröbner basis, see $[1,15]$.

The Gröbner basis is

$$
\begin{aligned}
& b_{1}\left(x_{0}, y_{0}\right)=d y_{0}, \\
& b_{2}\left(x_{0}, y_{0}\right)=\left(6 a x_{0}^{2}+5 \ell x_{0}^{4}\right) y_{0}+\left(6 a+10 \ell x_{0}^{2}\right) y_{0}^{3}+5 \ell y_{0}^{5}, \\
& b_{3}\left(x_{0}, y_{0}\right)=-8 d+6 a x_{0}^{3}+5 \ell x_{0}^{5}+\left(6 a x_{0}+10 \ell x_{0}^{3}\right) y_{0}^{2}+5 \ell x_{0} y_{0}^{4} .
\end{aligned}
$$

The only zero of $b_{1}\left(x_{0}, y_{0}\right)$ is $y_{0}^{0}=0$ since $d=\varepsilon^{-1} \delta>0$. So replacing $y_{0}^{0}$ into $b_{3}\left(x_{0}, y_{0}\right)$ and simplifying the new expressions, we reduce our problem to find a zero of the polynomial

$$
p\left(x_{0}\right)=\frac{1}{5 \ell} b_{3}\left(x_{0}, 0\right)=-\frac{8 d}{r \ell}+\frac{6 a}{5 \ell} x_{0}^{3}+x_{0}^{5} .
$$

As we know from algebra, there is no general formula to provide the roots of a quintic polynomial as polynomial (11). Nevertheless, some techniques may make this task easier. An elegant one can be found in [29]. In this paper, a way is provided to study the number of roots (complex and real, including multiplicity) of a polynomial of any order by only performing some calculations on the coefficients of the considered polynomial. We will present a brief summary of the algorithm of this method in the appendix.

By the Fundamental Theorem of Algebra, polynomial (11) has five roots taking into account their multiplicities and once we want to apply the averaging theory, we are only interested in the real simple roots of polynomial (11). Indeed, applying the method due to [29] and summarized 
in the appendix, we obtain

$$
\begin{aligned}
& D_{4}=\frac{384 a d^{2}}{\ell^{3}}, \\
& D_{5}=\frac{53747712}{78125} \frac{a^{5} d^{2}}{\ell^{7}}+20480 \frac{d^{4}}{\ell^{4}} .
\end{aligned}
$$

Therefore as described in the appendix, since $\alpha \lambda=\varepsilon^{2} a \ell<0$, by hypothesis we have that $D_{4}$ is negative. So polynomial (11) has a unique simple real root if $D_{5}=D=0$ according to statement (5) of the appendix. On the other hand, statement (3) says that polynomial (11) has three simple real roots if $D_{5}=D<0$. Moreover, $D$ and $D_{4}$ do not change when we replace $(a, \ell, d)$ by $\left(\varepsilon^{-1} \alpha, \varepsilon^{-1} \lambda, \varepsilon^{-1} \delta\right)$ using rescaling (5).

Now using the property on zeros of the Gröbner basis cited previously, the function $f\left(x_{0}, y_{0}\right)$ has a zero $\left(x_{0}^{0}, 0\right)$ if $D=0$ and three zeros $\left(x_{0}^{i}, 0\right)$ if $D<0$, with $i=1,2,3$. Besides the matrix $M=\partial\left(f_{1}, f_{2}\right) / \partial\left(x_{0}, y_{0}\right)$ evaluated at $y_{0}^{0}=0$ after rescaling $(5)$ is

$$
M=\frac{1}{\varepsilon}\left(\begin{array}{cc}
0 & \frac{\pi}{8}\left(6 \alpha x_{0}^{2}+5 \lambda x_{0}^{4}\right) \\
-\frac{\pi}{8}\left(18 \alpha x_{0}^{2}+25 \lambda x_{0}^{4}\right) & 0
\end{array}\right),
$$

whose determinant is

$$
\Pi\left(x_{0}\right)=\frac{1}{\varepsilon^{2}}\left(\frac{27}{16}\left(\alpha \pi x_{0}^{2}\right)^{2}+\frac{15}{4} \alpha \lambda\left(\pi x_{0}^{3}\right)^{2}+\frac{125}{64}\left(\lambda \pi x_{0}^{4}\right)^{2}\right) .
$$

Thus by computing a Gröbner basis for polynomials $\left\{\Pi\left(x_{0}\right), f_{1}\left(x_{0}, 0\right), f_{2}\left(x_{0}, 0\right)\right\}$ in the variables $\alpha, \delta, \lambda$ and $x_{0}$, we get the polynomials $\bar{b}_{1}\left(\alpha, \delta, \lambda, x_{0}\right)=\delta$ and $\bar{b}_{2}\left(\alpha, \delta, \lambda, x_{0}\right)=6 \alpha+5 \lambda x_{0}^{2}$. So $\bar{b}_{1}\left(\alpha, \delta, \lambda, x_{0}\right)>0$ because $\delta>0$ and it means that we cannot have $f_{1}, f_{2}$ and $\Pi$ equal to zero simultaneously. Consequently, $\Pi\left(x_{0}^{i}\right) \neq 0$ for all $i=0,1,2,3$. Then using the averaging theory we conclude the existence of a $2 \pi$-periodic solution if $D=0$ and three $2 \pi$-periodic solutions if $D<0$.

Now we exhibit the values of $\alpha, \lambda$ and $\delta$ for which system (4) has one or three $2 \pi$-periodic solutions. On the one hand, if consider $a=\frac{25}{18}, \ell=-1$ and $d=\frac{5}{12}$, we obtain $a \ell=-\frac{25}{18}<0$ and $D=0$. On the other hand, taking $d=18 a / 31$ and $\ell=-42 a / 155$, we get $a \ell=-42 a^{2} / 155<0$ and $D=-\frac{1425339825408}{823543}<0$. So Theorem 5 is proved.

We note that by assumption in Theorem 5 we have $a \ell<0$. So considering the notation of the appendix, the coefficients $D_{2}=-6 a / 5 \ell$ and $D_{3}=384 a d^{2} / \ell^{3}$ corresponding to polynomial (11) are positive and $D_{4}$ is negative. Thus the only possible configurations of roots of polynomial (11) are those one listed in statements (3) and (5) of the appendix. This fact means that we cannot have two or five periodic solutions when we consider $m=0, n_{2}=n_{3}=n_{4}=1$ and $n_{1}>1$.

Proof of Theorem 6 We start fixing the values $m=0, n_{1}=n_{2}=n_{4}=1$ and $n_{3}>1$. Now the vector field of system (8) is written as

$$
\left(y,-x+\varepsilon r y-\varepsilon r x^{2} y-\varepsilon a x^{3}-\varepsilon^{n_{3}} \ell x^{5}+\varepsilon d \cos t\right)^{\mathrm{T}},
$$


and then $F_{1}(t, \mathbf{x})=\left(0, r y-r x^{2} y-a x^{3}+d \cos t\right)$. With this expression of $F_{1}$, function (10) is as follows:

$$
\begin{aligned}
f_{1}\left(x_{0}, y_{0}\right)= & \int_{0}^{2 \pi}-(\sin t)\left(d \cos t+r\left(y_{0} \cos t-x_{0} \sin t\right)\right. \\
& \left.-r\left(y_{0} \cos t-x_{0} \sin t\right)\left(x_{0} \cos t+y_{0} \sin t\right)^{2}-a\left(x_{0} \cos t+y_{0} \sin t\right)^{3}\right) \mathrm{d} t \\
= & -\frac{1}{4} \pi\left(r x_{0}\left(-4+x_{0}^{2}+y_{0}^{2}\right)-3 a y_{0}\left(x_{0}^{2}+y_{0}^{2}\right)\right), \\
f_{2}\left(x_{0}, y_{0}\right)= & \int_{0}^{2 \pi}(\cos t)\left(d \cos t+r\left(y_{0} \cos t-x_{0} \sin t\right)-r\left(y_{0} \cos t-x_{0} \sin t\right)\left(x_{0} \cos t+y_{0} \sin t\right)^{2}\right. \\
& \left.-a\left(x_{0} \cos t+y_{0} \sin t\right)^{3}\right) \mathrm{d} t \\
= & \frac{1}{4} \pi\left(4 d-r y_{0}\left(-4+x_{0}^{2}+y_{0}^{2}\right)-3 a x_{0}\left(x_{0}^{2}+y_{0}^{2}\right)\right) .
\end{aligned}
$$

In order to find zeros $\left(x_{0}^{*}, y_{0}^{*}\right)$ of $f\left(x_{0}, y_{0}\right)$ as before, we compute a Gröbner basis $\left\{b_{k}\left(x_{0}, y_{0}\right)\right.$, $k=1, \ldots, 14\}$ in the variables $x_{0}$ and $y_{0}$ now for the set of polynomials $\left\{\bar{f}_{1}\left(x_{0}, y_{0}\right), \bar{f}_{2}\left(x_{0}, y_{0}\right)\right\}$ with $\bar{f}_{1,2}=\mp(4 / \pi) f_{1,2}$. We will look for zeros of two elements of the Gröbner basis for the polynomials $\left\{\bar{f}_{1}\left(x_{0}, y_{0}\right), \bar{f}_{2}\left(x_{0}, y_{0}\right)\right\}$ in the variables $x_{0}$ and $y_{0}$. These polynomials are

$$
\begin{aligned}
b_{1}\left(x_{0}, y_{0}\right)= & 144 a^{2} d r^{3}-4 d^{3} r^{3}+\left(108 a^{2} d^{2} r^{2}+144 a^{2} r^{4}-4 d^{2} r^{4}\right) y_{0} \\
& +144 a^{2} d r^{3} y_{0}^{2}+\left(81 a^{4} d^{2}+18 a^{2} d^{2} r^{2}+d^{2} r^{4}\right) y_{0}^{3}, \\
b_{2}\left(x_{0}, y_{0}\right)= & 216 a^{3} d r^{2}+\left(-324 a^{4} r^{2}-9 a^{2} d^{2} r^{2}+36 a^{2} r^{4}-d^{2} r^{4}\right) x_{0} \\
& +\left(27 a^{3} d^{2} r+216 a^{3} r^{3}+3 a d^{2} r^{3}\right) y_{0}+\left(243 a^{5} d+54 a^{3} d r^{2}+3 a d r^{4}\right) y_{0}^{2} .
\end{aligned}
$$

We must observe that $b_{1}\left(x_{0}, y_{0}\right)$ depends only on $y_{0}$. Then for each zero $y_{0}^{*}$ of $b_{1}\left(y_{0}\right)$, the second polynomial $b_{2}\left(x_{0}, y_{0}\right)$ provides a zero $x_{0}^{*}$ associated with $y_{0}^{*}$ because the coefficient of $x_{0}$ in $b_{2}\left(x_{0}, y_{0}\right)$ is not zero by the hypothesis. Now we will look for zeros of $b_{1}\left(y_{0}\right)$. Indeed, the discriminant $\Delta$ of the cubic polynomial $b_{1}\left(y_{0}\right)$ is written as

$$
\begin{aligned}
\Delta= & -16 d^{2} r^{6}\left(324 a^{4}+d^{2} r^{2}+9 a^{2}\left(d^{2}-4 r^{2}\right)\right)^{2} \\
& \times\left(2187 a^{4} d^{4}+27 d^{4} r^{4}-16 d^{2} r^{6}+18 a^{2}\left(27 d^{4} r^{2}-72 d^{2} r^{4}+32 r^{6}\right)\right) \\
= & -16 d^{2} r^{6} \Delta_{1}^{2} \Delta_{2} .
\end{aligned}
$$

So if $\Delta_{1}$ or $\Delta_{2}$ is zero, then $\Delta$ is zero and consequently $b_{1}\left(y_{0}\right)$ has a simple real root $y_{0}^{0}$ because this polynomial has no root with multiplicity 3 . Actually $b_{1}^{\prime \prime \prime}\left(y_{0}\right)=6\left(81 a^{4} d^{2}+18 a^{2} d^{2} r^{2}+\right.$ $\left.d^{2} r^{4}\right)>0$. The same occurs if $\Delta_{2}>0$, since this condition implies $\Delta<0$. On the other hand, if $\Delta_{2}<0$, then $\Delta>0$ and consequently polynomial $b_{1}\left(y_{0}\right)$ has three simple real roots $y_{0}^{i}$, $i=1,2,3$. Additionally, replacing each value $y_{0}^{i}$ into $b_{2}\left(x_{0}, y_{0}\right)$ we obtain the respective values $x_{0}^{i}$, for $=0,1,2,3$. We note that coming back through rescaling (5), the signs of $\Delta, \Delta_{1}$ and $\Delta_{2}$ does not change because each monomial composing $\Delta$ has the same degree.

Now we will verify the condition $M=\operatorname{det}\left((\partial f / \partial \mathbf{z})\left(x_{0}^{i}, y_{0}^{i}\right)\right) \neq 0$ for $i=0,1,2,3$. In fact $M$ is

$$
\frac{-\pi}{4 \varepsilon}\left(\begin{array}{cc}
-6 \alpha x_{0} y_{0}+\rho\left(-4+3 x_{0}^{2}+y_{0}^{2}\right) & 2 \rho x_{0} y_{0}-3 \alpha\left(x_{0}^{2}+3 y_{0}^{2}\right) \\
2 \rho x_{0} y_{0}+3 \alpha\left(3 x_{0}^{2}+y_{0}^{2}\right) & 6 \alpha x_{0} y_{0}+\rho\left(-4+x_{0}^{2}+3 y_{0}^{2}\right)
\end{array}\right),
$$

whose determinant $\Pi$ now is

$$
\Pi\left(x_{0}, y_{0}\right)=\frac{\pi^{2}}{16 \varepsilon^{2}}\left(27 \alpha^{2}\left(x_{0}^{2}+y_{0}^{2}\right)^{2}+\rho^{2}\left(-4+x_{0}^{2}+y_{0}^{2}\right)\left(-4+3 x_{0}^{2}+3 y_{0}^{2}\right)\right) .
$$


However for each $i=0,1,2,3$ the determinant $\Pi\left(x_{0}^{i}, y_{0}^{i}\right) \neq 0$ by hypothesis. Therefore, using the averaging theory described in Section 3, system (4) has a $2 \pi$-periodic solution if $\Delta_{1} \Delta_{2}=0$ or $\Delta_{2}>0$ and three $2 \pi$-periodic solutions if $\Delta_{2}<0$.

Now we present concrete values of $\alpha, \rho$ and $\delta$ for which we have one or three periodic solutions. Indeed taking $a=r=1$ and $d=6$, we have $\Delta_{2}=3452544>0$. Meantime if we consider $d=6 a$ and

$$
r=-\frac{9 a}{\sqrt{-9+4 \sqrt{6}}},
$$

we obtain $\Delta_{2}=-34012224 a^{8} /(9-4 \sqrt{6})^{2}<0$. We also observe that the values $(r, a, d)=$ $\left(\left(\frac{555}{4}\right) \sqrt{\frac{154073}{9622}}, 185,22\right)$ and $(r, a, d)=(585 \sqrt{3}, 195,585 \sqrt{2})$ make $\Delta_{1}$ and $\Delta_{2}$ equal to zero, respectively. This ends the proof of Theorem 6 .

Proof of Theorem 7 For this case, we assume $m=0$ and $n_{i}=1, i=1,2,3,4$. Then, the vector field of system (8) is written as

$$
\left(y,-x+\varepsilon r y-\varepsilon r x^{2} y-\varepsilon a x^{3}-\varepsilon \ell x^{5}+\varepsilon d \cos t\right)^{\mathrm{T}},
$$

and we have $F_{1}(t, \mathbf{x})=\left(0, r y-r x^{2} y-a x^{3}-\ell x^{5}+d \cos t\right)$. So it follows that

$$
\begin{aligned}
f_{1}\left(x_{0}, y_{0}\right)= & \int_{0}^{2 \pi}-(\sin t)\left(d \cos t+r\left(y_{0} \cos t-x_{0} \sin t\right)-r\left(y_{0} \cos t-x_{0} \sin t\right)\right. \\
& \left.\left(x_{0} \cos t+y_{0} \sin t\right)^{2}-a\left(x_{0} \cos t+y_{0} \sin t\right)^{3}-\ell\left(x_{0} \cos t+y_{0} \sin t\right)^{5}\right) \mathrm{d} t \\
= & -\frac{1}{8} \pi\left(2 r x_{0}\left(-4+x_{0}^{2}+y_{0}^{2}\right)-y_{0}\left(x_{0}^{2}+y_{0}^{2}\right)\left(6 a+5 \ell\left(x_{0}^{2}+y_{0}^{2}\right)\right),\right. \\
f_{2}\left(x_{0}, y_{0}\right)= & \int_{0}^{2 \pi}(\cos t)\left(d \cos t+r\left(y_{0} \cos t-x_{0} \sin t\right)-r\left(y_{0} \cos t-x_{0} \sin t\right)\right. \\
& \left.\left(x_{0} \cos t+y_{0} \sin t\right)^{2}-a\left(x_{0} \cos t+y_{0} \sin t\right)^{3}-\ell\left(x_{0} \cos t+y_{0} \sin t\right)^{5}\right) \mathrm{d} t \\
= & \frac{1}{8} \pi\left(8 d+8 r y_{0}-\left(x_{0}^{2}+y_{0}^{2}\right)\left(6 a x_{0}+2 r y_{0}+5 \ell x_{0}\left(x_{0}^{2}+y_{0}^{2}\right)\right)\right) .
\end{aligned}
$$

We will find zeros of $f\left(x_{0}, y_{0}\right)$ through the zeros of a Gröbner basis $\left\{b_{k}\left(x_{0}, y_{0}\right)\right\}$ of it. As before, instead of $f\left(x_{0}, y_{0}\right)$, the Gröbner basis will be related with the functions $\bar{f}_{1,2}=\mp(8 / \pi) f_{1,2}$.

One of the elements of the Gröbner basis in the variables $x_{0}$ and $y_{0}$ is

$$
\begin{aligned}
b_{1}\left(x_{0}, y_{0}\right)= & 144 a^{2} d r^{5}-4 d^{3} r^{5}+960 a d \ell r^{5}+1600 d \ell^{2} r^{5}+\left(108 a^{2} d^{2} r^{4}\right. \\
& +960 a d^{2} \ell r^{4}+2000 d^{2} \ell^{2} r^{4}+144 a^{2} r^{6}-4 d^{2} r^{6}+960 a \ell r^{6} \\
& \left.+1600 \ell^{2} r^{6}\right) y_{0}+\left(120 a d^{3} \ell r^{3}+500 d^{3} \ell^{2} r^{3}+144 a^{2} d r^{5}\right. \\
& \left.+1200 a d \ell r^{5}+2400 d \ell^{2} r^{5}\right) y_{0}^{2}+\left(81 a^{4} d^{2} r^{2}+540 a^{3} d^{2} \ell r^{2}+900 a^{2} d^{2} \ell^{2} r^{2}\right. \\
& \left.+18 a^{2} d^{2} r^{4}+300 a d^{2} \ell r^{4}+900 d^{2} \ell^{2} r^{4}+d^{2} r^{6}\right) y_{0}^{3} \\
& +\left(-450 a^{2} d^{3} \ell^{2} r-1500 a d^{3} \ell^{3} r+50 d^{3} \ell^{2} r^{3}\right) y_{0}^{4}+625 d^{4} \ell^{4} y_{0}^{5} .
\end{aligned}
$$

We note that again $b_{1}\left(x_{0}, y_{0}\right)$ depends only on $y_{0}$. Moreover, the Gröbner basis has another element $b_{2}\left(x_{0}, y_{0}\right)$ which is linear on $x_{0}$ and the coefficients depend on $y_{0}$, where the coefficient $C$ of $x_{0}$ is not zero by hypotheses. Its expression is too large and we will omit it here. It can be easily obtained by an algebraic manipulator. So for each zero $y_{0}^{*}$ of $b_{1}\left(y_{0}\right)$, the second polynomial $b_{2}\left(x_{0}, y_{0}\right)$ provides a zero $x_{0}^{*}$ associated with $y_{0}^{*}$. 
Now we will look for zeros of $b_{1}\left(y_{0}\right)$. Indeed using again the appendix, the conditions on $a$, $r, d$ and $\ell$ that provide three zeros, $\left(x_{0}^{i}, y_{0}^{i}\right)$, where $i=1,2,3$ for $b_{1}\left(y_{0}\right)$, are $D_{5}=-D_{5}^{1} D_{5}^{2} D<0$, where $D$ is given in Theorem 7 and $D_{5}^{1}$ and $D_{5}^{2}$ are the values

$$
\begin{aligned}
D_{5}^{1}= & \frac{110075314176 a^{8} r^{20}}{d^{20}\left(-9 a^{2}+r^{2}\right)^{28}}, \\
D_{5}^{2}= & \left(59049 a^{10} d^{4}+16 r^{14}+36 a^{2} r^{10}\left(11 d^{2}-4 r^{2}\right)-81 a^{4} r^{6}\left(d^{4}-12 d^{2} r^{2}+16 r^{4}\right)\right. \\
& \left.-6561 a^{8}\left(3 d^{4} r^{2}-4 d^{2} r^{4}\right)+729 a^{6}\left(3 d^{4} r^{4}-28 d^{2} r^{6}+16 r^{8}\right)\right)^{2} .
\end{aligned}
$$

By hypothesis $a r=\varepsilon^{-2} \alpha \rho \neq 0$ and $D_{5}^{2} \neq 0$, then $D_{5}^{1}$ and $D_{5}^{2}$ are positive. Therefore, in order to have $D_{5}$ negative, we must have $D$ positive. On the other hand, if $D_{5}$ is positive then according to statement (2) of the appendix, we have a unique zero $\left(x_{0}^{0}, y_{0}^{0}\right)$ for $b_{1}\left(y_{0}\right)$ because the value

$$
D_{2}=-\frac{1296 a^{2} r^{4}\left(-3 a^{2}+r^{2}\right)^{2}}{d^{2}\left(-9 a^{2}+r^{2}\right)^{4}}
$$

is negative if $\left(r^{2}-3 a^{2}\right)\left(r^{2}-9 a^{2}\right) \neq 0$. In addition by considering $a=\varepsilon^{-1} \alpha, r=\varepsilon^{-1} \rho$ and $d=$ $\varepsilon^{-1} \delta$, we get

$$
M=\frac{-\pi}{8 \varepsilon}\left(\begin{array}{cc}
2 \rho\left(-4+3 x_{0}^{2}+y_{0}^{2}\right)-\Gamma_{1} & \Gamma_{2}-\Gamma_{4}-5 \lambda \Gamma_{3}\left(x_{0}^{2}+5 y_{0}^{2}\right) \\
\Gamma_{2}+\Gamma_{4}+5 \lambda \Gamma_{3}\left(5 x_{0}^{2}+y_{0}^{2}\right) & 2 \rho\left(-4+x_{0}^{2}+3 y_{0}^{2}\right)-\Gamma_{1}
\end{array}\right),
$$

where $\Gamma_{1}=2 x_{0} y_{0}\left(3 \alpha+5 \ell\left(x_{0}^{2}+y_{0}^{2}\right)\right), \Gamma_{2}=-4 \rho x_{0} y_{0}, \Gamma_{3}=x_{0}^{2}+y_{0}^{2}$ and $\Gamma_{4}=6 \alpha\left(x_{0}^{2}+3 y_{0}^{2}\right)$. Moreover, determinant $\Pi$ of $M$ is written as

$$
\begin{aligned}
\Pi\left(x_{0}, y_{0}\right)= & \frac{1}{\varepsilon^{2}}\left(4 \rho^{2}\left(-4+x_{0}^{2}+y_{0}^{2}\right)\left(-4+3 x_{0}^{2}+3 y_{0}^{2}\right)\right. \\
& \left.+\left(x_{0}^{2}+y_{0}^{2}\right)^{2}\left(6 \alpha+\rho \lambda\left(x_{0}^{2}+y_{0}^{2}\right)\left(18 \alpha+25 \lambda x_{0}^{2}+3 y_{0}^{2}\right)\right)\right) .
\end{aligned}
$$

However, by hypothesis $\Pi\left(x_{0}^{i}, y_{0}^{i}\right) \neq 0$ for $i=0,1,2,3$ and hence we conclude the first part of Theorem 7.

Now we provide values for $\alpha, \rho, \lambda$ and $\delta$ for which we have one or three $2 \pi$-periodic solutions. First, we observe that taking $(a, r, d)=\left(1,2, \frac{8}{3}\right)$, we obtain

$$
D=-\frac{2770035802112}{6561}<0
$$

However, if we consider

$$
(a, r, d, \ell)=\left(\frac{1}{\sqrt{3}}, 1,1,-\frac{1}{(5 \sqrt{3})}\right)
$$

then $D=2752$. So Theorem 7 is proved.

Now we will prove that under the conditions $m=0$ and $n_{i}=1, i=1,2,3,4$, and the assumptions of Theorem 7, we cannot have two or five periodic solutions by using the averaging theory and rescaling (5). Indeed, we observe that the only possibility to have five periodic solutions is that statement (1) of the appendix holds. However, this condition does not hold since $D_{2}$ is negative for any $a, r$ and $d$ satisfying the hypotheses of Theorem 7 .

In addition, we note that the only condition that provides two periodic solutions according to the appendix is statement (7), and it needs $D_{5}=D_{4}=E_{2}=0$. Nevertheless, we start considering this condition and will find a Gröbner basis for the set of polynomials $\left\{D_{4}, D_{5}, E_{2}\right\}$ 
in the variables $a, d$ and $r$. First, we will consider the factor $D_{5}^{2}$ of $D_{5}$ because $D_{5}^{1} \neq 0$ by hypothesis. In this case, we obtain 55 polynomials in the Gröbner basis, where the first one is $g(d, r)=r^{62} \bar{g}(d, r)$ and

$$
\begin{aligned}
\bar{g}(d, r)= & 91125 d^{12}-438800 d^{10} r^{2}+919360 d^{8} r^{4}-1038464 d^{6} r^{6}+660992 d^{4} r^{8} \\
& -225280 d^{2} r^{10}+32768 r^{12} .
\end{aligned}
$$

Since $r \neq 0$ we must find a zero of $\bar{g}(d, r)$. So solving $\bar{g}(d, r)=0$ in the variable $r$ we obtain six pairs of values

$$
r_{ \pm}^{i}= \pm d \sqrt{q_{0}^{i}}
$$

where $i=1, \ldots, 6$ and for each $i$ the value $q_{0}^{i}$ is the $i$ th root of the polynomial

$$
q(x)=91125-438800 x+919360 x^{2}-1038464 x^{3}+660992 x^{4}-225280 x^{5}+32768 x^{6} .
$$

This polynomial has only complex roots. Indeed, if we study the function $q^{\prime}(x)$ which is given by

$$
q^{\prime}(x)=-438800+1838720 x-3115392 x^{2}+2643968 x^{3}-1126400 x^{4}+196608 x^{5},
$$

we can apply the method described in the appendix to show that the respective values $D_{2}$ and $D_{5}$ related to $q^{\prime}(x)$ are negative and positive, respectively. Therefore, $q^{\prime}(x)$ has only one real root $q_{0}^{\prime}$ whose approximate value is $q_{0}^{\prime}=\frac{106703}{100000}$. Additionally, the approximate value of $q(x)$ evaluated in $q_{0}^{\prime}$ is $q\left(q_{0}^{\prime}\right)=\frac{16558687}{10000}>0$. So if we observe that the coefficient of $x^{6}$ is positive, it follows that the minimum value of $q(x)$ is positive. Then each $r_{ \pm}^{i}, i=1, \ldots, 6$, is a complex value and the correspondent Gröbner basis has no zeros. Then we cannot have a common real zero of $D_{4}$, $D_{5}^{2}$ and $E_{2}$ and so statement (7) in the appendix does not hold. Consequently, it is not possible to obtain two periodic solutions. The proof considering the factor $D$ of $D_{5}$ instead of $D_{5}^{2}$ leads to the same polynomial $g(d, r)$ and then we have the same conclusion.

Proof of Theorem 8 In what follows we take $m=0, n_{1}=n_{3}=n_{4}=1$ and $n_{2}>1$. So the vector field of system (8) becomes

$$
\left(y,-x+\varepsilon r y-\varepsilon r x^{2} y-\varepsilon^{n_{2}} a x^{3}-\varepsilon \ell x^{5}+\varepsilon d \cos t\right)^{\mathrm{T}},
$$

and we obtain $F_{1}(t, \mathbf{x})=\left(0, r y-r x^{2} y-\ell x^{5}+d \cos t\right)$. So $f\left(x_{0}, y_{0}\right)$ is written as

$$
\begin{aligned}
f_{1}\left(x_{0}, y_{0}\right)= & \int_{0}^{2 \pi}-(\sin t)\left(d \cos t+r\left(y_{0} \cos t-x_{0} \sin t\right)-r\left(y_{0} \cos t-x_{0} \sin t\right)\right. \\
& \left.\left(x_{0} \cos t+y_{0} \sin t\right)^{2}-\ell\left(x_{0} \cos t+y_{0} \sin t\right)^{5}\right) \mathrm{d} t \\
= & -\frac{1}{8} \pi\left(2 r x_{0}\left(-4+x_{0}^{2}+y_{0}^{2}\right)-5 \ell y_{0}\left(x_{0}^{2}+y_{0}^{2}\right)^{2}\right), \\
f_{2}\left(x_{0}, y_{0}\right)= & \int_{0}^{2 \pi}(\cos t)\left(d \cos t+r\left(y_{0} \cos t-x_{0} \sin t\right)-r\left(y_{0} \cos t-x_{0} \sin t\right)\right. \\
& \left.\left(x_{0} \cos t+y_{0} \sin t\right)^{2}-\ell\left(x_{0} \cos t+y_{0} \sin t\right)^{5}\right) \mathrm{d} t \\
= & \frac{1}{8} \pi\left(8 d-2 r y_{0}\left(-4+x_{0}^{2}+y_{0}^{2}\right)-5 \ell x_{0}\left(x_{0}^{2}+y_{0}^{2}\right)^{2}\right) .
\end{aligned}
$$

Again we will find zeros of $f\left(x_{0}, y_{0}\right)$ through the roots of a Gröbner basis $\left\{b_{k}\left(x_{0}, y_{0}\right)\right\}$ of $f\left(x_{0}, y_{0}\right)$. We will find a Gröbner basis of the functions $\bar{f}_{1,2}=\mp(8 / \pi) f_{1,2}$. 
An element of the Gröbner basis in the variables $x_{0}$ and $y_{0}$ is

$$
\begin{aligned}
b_{1}\left(x_{0}, y_{0}\right)= & -4 d^{3} r^{5}+1600 d L^{2} r^{5}+\left(2000 d^{2} \ell^{2} r^{4}-4 d^{2} r^{6}+1600 L^{2} R^{6}\right) y_{0} \\
& +\left(500 d^{3} \ell^{2} r^{3}+2400 d \ell^{2} r^{5}\right) y_{0}^{2}+\left(900 d^{2} \ell^{2} r^{4}+d^{2} r^{6}\right) y_{0}^{3}+50 d^{3} \ell^{2} r^{3} y_{0}^{4}+625 d^{4} \ell^{4} y_{0}^{5} .
\end{aligned}
$$

As before, $b_{1}\left(x_{0}, y_{0}\right)$ depends only on $y_{0}$ and another element $b_{2}\left(x_{0}, y_{0}\right)$ of the Gröbner basis is linear on $x_{0}$ with coefficients depending on $y_{0}$, where the coefficient $C$ of $x_{0}$ in $b_{2}\left(x_{0}, y_{0}\right)$ is not zero by hypotheses. We will not present the expression of $b_{2}\left(x_{0}, y_{0}\right)$ in order to avoid large expressions. Then for each zero $y_{0}^{*}$ of $b_{1}\left(y_{0}\right)$ we have a second zero $x_{0}^{*}$ through $b_{2}\left(x_{0}, y_{0}\right)$ related to $y_{0}^{*}$.

In order to apply the method described in [29] and the appendix for $b_{1}\left(y_{0}\right)$, we will perform the translation $y_{0}=\varphi-\left(2 r^{3} / d(125 \ell)^{2}\right)$. With this translation we obtain a new polynomial $b_{1}^{*}$ in the form

$$
b_{1}^{*}(\varphi)=\varphi^{5}+\frac{4500 \ell^{2} r^{4}-3 r^{6}}{3125 d^{2} \ell^{4}} \varphi^{3}+\frac{2 k_{1}}{\bar{k}_{1} d^{3} \ell^{6}} \varphi^{2}+\frac{4 r^{4} k_{2}}{\bar{k}_{2} d^{4} \ell^{8}} \varphi-\frac{4 r^{5} k_{3}}{\bar{k}_{3} d^{5} \ell^{10}},
$$

where

$$
\begin{aligned}
k_{1}= & 156250 d^{2} \ell^{4} r^{3}+750000 \ell^{4} r^{5}-13500 \ell^{2} r^{7}+r^{9}, \\
\bar{k}_{1}= & 390625, \\
k_{2}= & 39062500 d^{2} \ell^{6}-390625 \ell^{4}\left(d^{2}-80 \ell^{2}\right) r^{2}-1500000 \ell^{4} r^{4}+13500 \ell^{2} r^{6}+3 r^{8}, \\
\bar{k}_{2}= & 48828125, \\
k_{3}= & 48828125 d^{4} \ell^{6}-781250 d^{2}\left(25000 \ell^{8}-500 \ell^{6} r^{2}+3 \ell^{4} r^{4}\right)+2 r^{4} \\
& \left(156250000 \ell^{6}-3750000 \ell^{4} r^{2}+22500 \ell^{2} r^{4}+9 r^{6}\right), \\
\bar{k}_{3}= & 30517578125 .
\end{aligned}
$$

We observe that the translation performed does not change the number or kind of the zeros of the original polynomial $b_{1}$. Now we will apply the method of the appendix for $b_{1}^{*}$. Indeed, we have

$$
\begin{aligned}
& D_{2}=N_{2}, \\
& D_{3}=-\frac{48 r^{6}}{9765625 d^{6} \ell^{10}} N_{3}, \\
& D_{4}=-\frac{16 r^{12}}{3814697265625 d^{12} \ell^{18}} N_{4}, \\
& D_{5}=\frac{256 r^{20}}{1490116119384765625 d^{20} \ell^{26}} N_{5} M_{5} .
\end{aligned}
$$

Therefore, if $D_{5}$ is negative then $b_{1}^{*}$ and consequently $b_{1}$ have exactly three zeros, and hence function $f=\left(f_{1}, f_{2}\right)$ has exactly three zeros $\left(x_{0}^{i}, y_{0}^{i}\right), i=1,2,3$. On the other hand, if $D_{5}$ is positive and one of the values $D_{2}, D_{3}$ or $D_{4}$ is non-positive, then $f$ has exactly one zero $\left(x_{0}^{0}, y_{0}^{0}\right)$. Moreover, using rescaling (5) the matrix $M$ is the same as the one of the proof of Theorem 7 taking $a=0$. In addition, determinant $\Pi$ of $M$ is written as

$$
\Pi\left(x_{0}, y_{0}\right)=\frac{1}{\varepsilon^{2}}\left(125 \lambda^{2}\left(x_{0}^{2}+y_{0}^{4}\right)+4 \rho^{2}\left(-4+x_{0}^{2}+y_{0}^{2}\right)\left(-4+3 x_{0}^{2}+3 y_{0}^{2}\right)\right) .
$$

By hypothesis $\Pi\left(x_{0}^{i}, y_{0}^{i}\right) \neq 0$ for $i=0,1,2,3$, then we have the first part of Theorem 8 proved. 
Now we exhibit values of $\rho, \lambda$ and $\delta$ for which we have either one or three periodic solutions. First, we consider

$$
(r, \ell, d)=\left(\sqrt{31}, 1, \frac{1}{125} \sqrt{1547875-226851 \sqrt{35}}\right)
$$

With these values we obtain

$$
\begin{aligned}
& N_{3}=\frac{1}{625}(206837701817900-10419995302263 \sqrt{35}), \\
& N_{5}=\frac{2(-649491478051728715458244+109639832554794027558525 \sqrt{35})}{48828125},
\end{aligned}
$$

which are positive. On the other hand, considering $(r, \ell, d)=(\sqrt{31} / 4,1,1)$ we get $N_{5}=$ $-\frac{258261575}{1048576}<0$. This ends the proof of Theorem 8 .

Now we will show that if $m=0, n_{1}=n_{3}=n_{4}=1$ and $n_{2}>1$, then we cannot have two periodic solutions by using the averaging theory described in Section 3 and rescaling (5). Indeed in order that function $b_{1}^{*}(\varphi)$, given in the proof of Theorem 8 , has two periodic solutions, we need statement (7) of the appendix. We start considering the conditions $N_{5}=N_{4}=E_{2}=0$ and will see that these equalities imply that $N_{3}$ cannot be negative. We claim that $N_{5}$ and $M_{5}$ are factors of $D_{5}$ as we saw in the last proof. Also we will not present the expression of $E_{2}$ in order to avoid its large expression. It can be obtained by an algebraic manipulator such as Mathematica. Additionally, we note that $N_{4}=N_{5}=0$ implies $D_{4}=D_{5}=0$, which are necessary conditions for having exactly two real roots.

We start replacing the condition $N_{4}=N_{5}=E_{2}=0$ for other ones easier when $r>0$. It is easy to see that if $r=0$ we have $N_{3}=0$ and consequently we cannot get two roots for $b_{1}^{*}$. Now we obtain a Gröbner basis for the set of polynomials $\left\{N_{4}, N_{5}, E_{2}\right\}$ in the variables $d, r$ and $\ell$. This basis has 12 elements, where 2 of them are

$$
\begin{aligned}
g_{1}(r, \ell)= & 125 \cdot 10^{8} \ell^{8}+2 \cdot 10^{8} \ell^{6} r^{2}-100000 \ell^{4} r^{4}-10400 \ell^{2} r^{6}-3 r^{8}, \\
g_{2}(d, r, \ell)= & 25 d^{4} \ell^{2}-110000 d^{2} \ell^{4}-4000000 \ell^{6}-1400 d^{2} \ell^{2} r^{2}+80000 \ell^{4} r^{2} \\
& -3 d^{2} r^{4}+1200 \ell^{2} r^{4} .
\end{aligned}
$$

In addition, the resultant of these two polynomials with respect to the variable $r$ has the factor

$$
g_{3}(d, \ell)=d^{8}-1240000 d^{6} \ell^{2}-269440000 d^{4} \ell^{4}+6144 \cdot 10^{6} d^{2} \ell^{6}+28672 \cdot 10^{8} \ell^{8} .
$$

Consequently, we will replace the problem of finding a zero of $N_{4}, N_{5}$ and $E_{2}$ by the problem of finding a zero of $g_{1}, g_{2}$ and $g_{3}$. Moreover, we suppose that we have a common zero between each $g_{i}$ and $N_{3}, i=1,2,3$. With this supposition and computing the resultant between $g_{1}$ and $N_{3}$, we obtain a new polynomial $g_{4}$ in the variables $r$ and $d$ given by

$$
\begin{aligned}
g_{4}(r, d)= & 1423828125 d^{16}+25974000000 d^{14} r^{2}-47933388000000 d^{12} r^{4} \\
& -478810245120000 d^{10} r^{6}+1711832243200000 d^{8} r^{8} \\
& -125591060860108800 d^{6} r^{10}-1334616713986048000 d^{4} r^{12} \\
& +5191865574606503936 d^{2} r^{14}-4405603330689073152 r^{16}
\end{aligned}
$$

However, the resultant between $g_{3}$ and $g_{4}$ is written as $K r^{128}$, where $K$ is a positive constant. This polynomial cannot be zero since $r \geq 0$. It means that when we consider each $g_{i}$ zero, $N_{3}$ must be 
positive or negative, exclusively. But taking the values $(r, \ell, d)=\left(r_{0}, \ell_{0}, 1\right)$ we have $g_{i} \equiv 0$, for each $i=1,2,3$ and $N_{3}$ is approximately $\frac{8738483}{2500}>0$, where $r_{0}$ and $\ell_{0}$ are roots of the polynomials

$$
\bar{r}(x)=-125-44400 x^{2}+184640 x^{4}-237568 x^{6}+86016 x^{8},
$$

and

$$
\bar{l}(x)=1-1240000 x^{2}-269440000 x^{4}+6144000000 x^{6}+2867200000000 x^{8},
$$

respectively. So $N_{3}$ is positive when $g_{i} \equiv 0$, for each $i=1,2,3$, and then $D_{3}$ is negative. Hence using the factor $N_{5}$ of $D_{5}$ we cannot obtain two periodic solutions. The proof that cannot exists two periodic solutions for the case that we consider the factor $M_{5}$ of $D_{5}$ instead of $N_{5}$ is similar and we will omit it here.

We remark that we cannot prove analytically the non-existence of five periodic solutions for system (8) considering $m=0, n_{1}=n_{3}=n_{4}=1$ and $n_{2}>1$. Actually, using the algebraic manipulator Mathematica, we obtained evidences that this number of periodic solutions cannot happen, but an analytical treatment is not trivial.

\subsection{Discussion of the results}

Now we discuss some aspects of the averaging method presented in Section 3 in order to find periodic solutions in system (4). In fact by using the averaging theory and rescaling (5), we shall see that we cannot prove that system (4) has periodic solutions except in the cases presented in Theorems 1-8.

We start studying the possible values to the powers of $\varepsilon$ in system (8). This is important because these powers play an important role in the averaging theory described in Section 3 because they determine the terms of order 1 that we are interested in. Indeed, we note that each one of the five different powers of $\varepsilon$ in system (8) must be non-negative. Therefore, considering these powers as zero or positive, we have 32 possible combinations of these 5 different powers of $\varepsilon$. Actually some of them are not algebraically possible. Table 1 exhibits only the possible case.

Observing Table 1 we see that in fact we have only 18 cases. Moreover, in order to apply the averaging theory for one of the 18 cases, we must integrate the equations of the non-perturbed part of the vector field of system (8). This is not a simple task because the major part of these equations are non-linear and non-autonomous. Indeed in each case from 1 to 8 and from 11 to 16 we could not integrate the equations even using the algebraic manipulator Mathematica or Maple. The non-integrable cases from Table 1 are listed as follows:

$$
\begin{aligned}
& \text { Case 1: } F_{0}(t, x)=\left(y,-x+r y-a x^{3}-r x^{2} y-\ell x^{5}+d \cos t\right), \\
& \text { Case 2: } F_{0}(t, x)=\left(y,-x+r y-a x^{3}-r x^{2} y-\ell x^{5}\right), \\
& \text { Case 3: } F_{0}(t, x)=\left(y,-x+r y-a x^{3}-r x^{2} y+d \cos t\right), \\
& \text { Case 4: } F_{0}(t, x)=\left(y,-x+r y-a x^{3}-r x^{2} y\right), \\
& \text { Case 5: } F_{0}(t, x)=\left(y,-x+r y-r x^{2} y-\ell x^{5}+d \cos t\right), \\
& \text { Case 6: } F_{0}(t, x)=\left(y,-x+r y-r x^{2} y-\ell x^{5}\right), \\
& \text { Case 7: } F_{0}(t, x)=\left(y,-x+r y-r x^{2} y+d \cos t\right), \\
& \text { Case 8: } F_{0}(t, x)=\left(y,-x+r y-r x^{2} y\right), \\
& \text { Case 11: } F_{0}(t, x)=\left(y,-x-a x^{3}-\ell x^{5}+d \cos t\right), \\
& \text { Case 12: } F_{0}(t, x)=\left(y,-x-a x^{3}-\ell x^{5}\right), \\
& \text { Case 13: } F_{0}(t, x)=\left(y,-x-a x^{3}+d \cos t\right), \\
& \text { Case 14: } F_{0}(t, x)=\left(y,-x-a x^{3}\right), \\
& \text { Case 15: } F_{0}(t, x)=\left(y,-x-\ell x^{5}+d \cos t\right), \\
& \text { Case 16: } F_{0}(t, x)=\left(y,-x-\ell x^{5}\right) .
\end{aligned}
$$


Table 1. Here, when appear $\varnothing$, this means that the corresponding cases cannot occur.

\begin{tabular}{|c|c|c|c|c|c|}
\hline & Possible & ation of powers & system (9) & & \\
\hline \multirow[t]{12}{*}{$n_{1}=0$} & $2 m+n_{1}=0$ & $2 m+n_{2}=0$ & $4 m+n_{3}=0$ & $-m+n_{4}=0$ & $\mathrm{C} 1$ \\
\hline & & & & $-m+n_{4}>0$ & $\mathrm{C} 2$ \\
\hline & & & $4 m+n_{3}>0$ & $-m+n_{4}=0$ & $\mathrm{C} 3$ \\
\hline & & & & $-m+n_{4}>0$ & $\mathrm{C} 4$ \\
\hline & & $2 m+n_{2}>0$ & $4 m+n_{3}=0$ & $-m+n_{4}=0$ & C5 \\
\hline & & & & $-m+n_{4}>0$ & C6 \\
\hline & & & $4 m+n_{3}>0$ & $-m+n_{4}=0$ & $\mathrm{C} 7$ \\
\hline & & & & $-m+n_{4}>0$ & $\mathrm{C} 8$ \\
\hline & $2 m+n_{1}>0$ & $2 m+n_{2}=0$ & & $\varnothing$ & \\
\hline & & $2 m+n_{2}>0$ & $4 m+n_{3}=0$ & $\varnothing$ & \\
\hline & & & $4 m+n_{3}>0$ & $-m+n_{4}=0$ & C9 \\
\hline & & & & $-m+n_{4}>0$ & $\mathrm{C} 10$ \\
\hline \multirow[t]{9}{*}{$n_{1}>0$} & $2 m+n_{1}=0$ & & $\varnothing$ & & \\
\hline & $2 m+n_{1}>0$ & $2 m+n_{2}=0$ & $4 m+n_{3}=0$ & $-m+n_{4}=0$ & C11 \\
\hline & & & & $-m+n_{4}>0$ & $\mathrm{C} 12$ \\
\hline & & & $4 m+n_{3}>0$ & $-m+n_{4}=0$ & C13 \\
\hline & & & & $-m+n_{4}>0$ & C14 \\
\hline & & $2 m+n_{2}>0$ & $4 m+n_{3}=0$ & $-m+n_{4}=0$ & $\mathrm{C} 15$ \\
\hline & & & & $-m+n_{4}>0$ & $\mathrm{C} 16$ \\
\hline & & & $4 m+n_{3}>0$ & $-m+n_{4}=0$ & C17 \\
\hline & & & & $-m+n_{4}>0$ & $\mathrm{C} 18$ \\
\hline
\end{tabular}

Note: The notation $\mathrm{C} i, i=1, \ldots, 18$ enumerate each possible case.

In particular, we observe that cases 12, 14 and 16 turn the non-perturbed part of system (8) into a Hamiltonian system. In [3], Buica and Llibre provide a method to apply the averaging theory in planar systems when the system is Hamiltonian, but the expressions become also too much complicated and again we could not integrate the equations in cases 12, 14 and 16.

In cases 9, 10 and 17, we can integrate the expressions but in these cases the hypotheses of the averaging method do not apply. In these cases, we have the following expressions for $F_{0}(t, \mathbf{x})$.

Case 9: $F_{0}(t, \mathbf{x})=(y,-x+r y+d \cos t)$,

Case 10: $F_{0}(t, \mathbf{x})=(y,-x+r y)$,

Case 17: $F_{0}(t, \mathbf{x})=(y,-x+d \cos t)$.

Solution $x(t)$ in case 9 is

$$
C^{-} \mathrm{e}^{(1 / 2) t\left(r-\sqrt{-4+r^{2}}\right)}+C^{+} \mathrm{e}^{(1 / 2) t\left(r+\sqrt{-4+r^{2}}\right)}-\left(\frac{d}{r}\right) \sin t,
$$

where

$$
C^{\mp}=\frac{\mp 2 d+r\left(\left( \pm r+\sqrt{-4+r^{2}}\right) x_{0} \mp 2 y_{0}\right)}{2 r \sqrt{-4+r^{2}}} .
$$

In order that $x(t)$ be periodic, we must have $C^{-}$and $C^{+}$equal to zero. But these conditions are verified only if we take $x_{0}=0$ and $y_{0}=-d / r$. So $x_{0}$ and $y_{0}$ are fixed and then the unperturbed system $\dot{x}=F_{0}(t, x)$ has no sub-manifold of periodic solutions. Consequently, we cannot apply the averaging theory in this case.

On the other hand, in case 10 solution $x(t)$ is written as

$$
C^{-} \mathrm{e}^{(1 / 2) t\left(r-\sqrt{-4+r^{2}}\right)}+C^{+} \mathrm{e}^{(1 / 2) t\left(r+\sqrt{-4+r^{2}}\right)}-\left(\frac{d}{r}\right) \sin t,
$$


where

$$
C^{\mp}=\frac{\left( \pm r+\sqrt{-4+r^{2}}\right) x_{0} \mp 2 y_{0}}{2 r \sqrt{-4+r^{2}}} .
$$

Again we need to choose $C^{-}$and $C^{+}$equal to zero, but this happens only if $\left(x_{0}, y_{0}\right)=(0,0)$. So we cannot apply the averaging theory because $(0,0)$ is the equilibrium point of the non-perturbed part of system (8) in case 10.

Finally, in case 17 solutions $x(t)$ and $y(t)$ have the form

$$
\begin{aligned}
& x(t)=x_{0} \cos t+y_{0} \sin t+\frac{d}{2} t \cos t, \\
& y(t)=y_{0} \cos t-x_{0} \sin t+\frac{d}{2}(t \cos t+\sin t) .
\end{aligned}
$$

It is immediate to note that these solutions are non-periodic because $d$ is positive.

Case 18 provides positive results and Theorems 1-8 are based on this case. In fact, we observe that in case 18 the vector field of system (8) is

$$
\left(y,-x+\varepsilon^{n_{1}} r y-\varepsilon^{2 m+n_{1}} r x^{2} y-\varepsilon^{2 m+n_{2}} a x^{3}-\varepsilon^{4 m+n_{3}} \ell x^{5}+\varepsilon^{-m+n_{4}} d \cos t\right)^{\mathrm{T}} .
$$

As we commented before, the periodic solutions of our problem correspond to the simple zeros of function (10). So in order to calculate the zeros of the function $f(\mathbf{z})$ given in Equation (10), we must determine $F_{1}(t, \mathbf{x})$, where $F_{1}(t, \mathbf{x})$ is determined by the terms of order 1 on $\varepsilon$ of the vector field of system (8). However, we see that the expression of $F_{1}(t, \mathbf{x})$ depends on the values $m$ and $n_{i}, i=1,2,3,4$. In fact, if $m$ is positive and observing condition (7), the only terms of the vector field of order 1 on $\varepsilon$ can be generated by powers $n_{1}$ and $-m+n_{4}$, where these two values can be 1 or greater than 1 . This implies four possibilities. On the other hand, if $m$ is zero the powers of $\varepsilon$ in the vector field of system (8) depend on $n_{1}, n_{2}, n_{3}$ and $n_{4}$. Again, each one of these powers can be 1 greater than 1 . So, if $m$ is zero we have 16 possibilities for $F_{1}$. Then case 18 has 20 subcases corresponding to the different possibilities of $F_{1}(t, \mathbf{x})$. These subcases are presented in Table 2 .

Table 2. Possible expressions for $F_{1}(t, \mathbf{x})$ when $m^{2}+n_{2}^{2}, m^{2}+n_{3}^{2}, n_{4}-m$ and $n_{1}$ are positives.

\begin{tabular}{lll}
\hline Sub. & Conditions & Second coordinate of $F_{1}(t, \mathbf{x})$ \\
\hline 1 & $m=0, n_{i}=1, i=1,2,3,4$ & $r y-a x^{3}-r x^{2} y-\ell x^{5}+d \cos t$ \\
2 & $m=0, n_{i}=1, i=1,2,3, n_{4}>1$ & $r y-a x^{3}-r x^{2} y-\ell x^{5}$ \\
3 & $m=0, n_{i}=1, i=1,2,4, n_{3}>1$ & $r y-a x^{3}-r x^{2} y+d \cos t$ \\
4 & $m=0, n_{i}=1, i=1,3,4, n_{2}>1$ & $r y-r x^{2} y-\ell x^{5}+d \cos t$ \\
5 & $m=0, n_{i}=1, i=2,3,4, n_{1}>1$ & $-a x^{3}-\ell x^{5}+d \cos t$ \\
6 & $m=0, n_{1}=n_{2}=1, n_{3}, n_{4}>1$ & $r y-a x^{3}-r x^{2} y$ \\
7 & $m=0, n_{1}=n_{3}=1, n_{2}, n_{4}>1$ & $r y-r x^{2} y-\ell x^{5}$ \\
8 & $m=0, n_{1}=n_{4}=1, n_{2}, n_{3}>1$ & $r y-r x^{2} y+d \cos t$ \\
9 & $m=0, n_{2}=n_{3}=1, n_{1}, n_{4}>1$ & $-a x^{3}-\ell x^{5}$ \\
10 & $m=0, n_{2}=n_{4}=1, n_{1}, n_{3}>1$ & $-a x^{3}+d \cos t$ \\
11 & $m=0, n_{3}=n_{4}=1, n_{1}, n_{2}>1$ & $-\ell x^{5}+d \cos t$ \\
12 & $m=0, n_{1}=1, n_{i}>1, i=2,3,4$ & $r y-r x^{2} y$ \\
13 & $m=0, n_{2}=1, n_{i}>1, i=1,3,4$ & $-a x^{3}$ \\
14 & $m=0, n_{3}=1, n_{i}>1, i=1,2,4$ & $-\ell x^{5}$ \\
15 & $m>0, n_{4}=1, n_{i}>1, i=1,2,3$ & $d \cos t$ \\
16 & $m>0, n_{i}>1, i=1,2,3,4$ & 0 \\
17 & $m>0, n_{1}=1,-m+n_{4}=1$ & $r y+d \cos t$ \\
18 & $m>0, n_{1}=1,-m+n_{4}>1$ & $r y$ \\
19 & $m>0, n_{1}>1,-m+n_{4}=1$ & $d \cos t$ \\
20 & $m>0, n_{1}>1,-m+n_{4}>1$ & 0 \\
\hline
\end{tabular}

Notes: We exhibit only the second coordinate of $F_{1}(t, \mathbf{x})$ because the first one has no terms depending on $\varepsilon$. 
Theorems 1-8 correspond to subcases $8,10,11,17,5,3,1$ and 4 , respectively. These are the only subcases of case 18 where the averaging method provides positive results. Indeed, in cases 15, 16, 19 and 20, function (10) does not have zeros. On the other hand, in cases 2, 6, 7, 13, 14, 18 , the only zero of function $(10)$ is $\left(x_{0}, y_{0}\right)=(0,0)$ that corresponds to the equilibrium point of the system, and consequently, in these subcases the system does not have periodic solutions. In cases 9 and 12, function (10) has real zeros different from $(0,0)$, but they are non-isolated and then the Jacobian of function (10) at the zero is zero, and consequently, the averaging theory cannot be applied.

\section{The averaging theory for periodic solutions}

Now we present the basic results on the averaging theory of first order that we need to prove our results.

Consider the problem of bifurcation of $T$-periodic solutions from differential systems of the form

$$
\dot{\mathbf{x}}=F_{0}(t, \mathbf{x})+\varepsilon F_{1}(t, \mathbf{x})+\varepsilon^{2} R(t, \mathbf{x}, \varepsilon),
$$

with $\varepsilon=0$ to $\varepsilon \neq 0$ sufficiently small. Here the functions $F_{0}, F_{1}: \mathbb{R} \times \Omega \rightarrow \mathbb{R}^{n}$ and $R: \mathbb{R} \times$ $\Omega \times\left(-\varepsilon_{f}, \varepsilon_{f}\right) \rightarrow \mathbb{R}^{n}$ are $\mathcal{C}^{2}, T$-periodics in the first variable and $\Omega$ is an open subset of $\mathbb{R}^{n}$. One of the main assumptions is that the unperturbed system

$$
\dot{\mathbf{x}}=F_{0}(t, \mathbf{x})
$$

has a manifold of periodic solutions. A solution to this problem is given using the averaging theory.

Indeed, assume that there is an open set $V$ with $\bar{V} \subset D \subset \Omega$ and such that for each $\mathbf{z} \in \bar{V}$, $\mathbf{x}(\cdot, \mathbf{z}, 0)$ is $T$-periodic, where $\mathbf{x}(\cdot, \mathbf{z}, 0)$ is the solution of the unperturbed system (13) with $\mathbf{x}(0)=$ z. Answer to the problem of bifurcation of $T$-periodic solutions from $\mathbf{x}(\cdot, \mathbf{z}, 0)$ is given in the following theorem.

THEOREM 9 We assume that there exists an open set $V$ with $\bar{V} \subset D$ and such that for each $z \in \bar{V}, \boldsymbol{x}(\cdot, z, 0)$ is T-periodic and consider the function $f: \bar{V} \rightarrow \mathbb{R}^{n}$ given by

$$
f(z)=\int_{0}^{\mathrm{T}} Y^{-1}(t, z) F_{1}(t, x(t, z, 0)) \mathrm{d} t .
$$

Then the following statements hold.

(a) If there exists $a \in V$ with $f(a)=0$ and $\operatorname{det}((\partial f / \partial z)(a)) \neq 0$, then there exists a $T$-periodic solution $\varphi(\cdot, \varepsilon)$ of system $(12)$ such that $\varphi(0, \varepsilon) \rightarrow$ a as $\varepsilon \rightarrow 0$.

(b) The type of stability of the periodic solution $\varphi(\cdot, \varepsilon)$ is given by the eigenvalues of the Jacobian matrix $M=((\partial f / \partial z)(a))$.

For a proof of Theorem 9(a), see [4, Corollary 1].

In fact, the result of Theorem 9 is a classical result due to Malkin [20] and Roseau [21]. For a shorter proof of Theorem 9(a), see [4].

For additional information on the averaging theory, see [23].

\section{Disclosure statement}

No potential conflict of interest was reported by the authors. 


\section{Funding}

The first author is supported by the FAPESP-BRAZIL grants 2010/18015-6, 2012/05635-1 and 2013/25828-1. The second author is partially supported by MINECO/FEDER grants MTM2008-03437 and MTM2013-40998-P, an AGAUR grant number 2014SGR568, an ICREA Academia, grants FP7-PEOPLE-2012-IRSES 318999 and 316338 , FEDER-UNAB-10-4E-378 and a CAPES grant 88881. 030454/2013-01 do Programa CSF-PVE.

\section{References}

[1] W.W. Adams and P. Loustaunau, An Introduction to Gröbner Bases, Graduate Studies in Mathematics, Vol. 3, American Mathematical Society, Providence, RI, 1994.

[2] K. Bod, C. Edwards, J. Guckenheimer, S. Guharay, K. Hoffman, J. Hubbard, R. Oliva, and W. Weckesser, The forced Van der Pol equation II: Canards in the reduced system, SIAM J. Appl. Dyn. Syst. 2 (2003), pp. 570-608.

[3] A. Buica and J. Llibre, Averaging methods for finding periodic orbits via Brouwer degree, Bull. Sci. Math. 128 (2004), pp. 7-22.

[4] A. Buica, J.P. Françoise, and J. Llibre, Periodic solutions of nonlinear periodic differential systems with a small parameter, Comm. Pure Appl. Anal. 6 (2007), pp. 103-111.

[5] A. Chen and G. Jiang, Periodic solution of the Duffing-Van der Pol oscillator by homotopy perturbation method, Int. J. Comput. Math. 87 (2010), pp. 2688-2696.

[6] Y.M. Chen and J.K. Liu, Uniformly valid solution of limit cycle of the Duffing-van der Pol equation, Mech. Res. Comm. 36 (2009), pp. 845-850.

[7] W.O. Criminale, T.L. Jackson, and P.W. Nelson, Limit cycle-strange attractor competition, Stud. Appl. Math. 112 (2004), pp. 133-160.

[8] C. Egami and N. Hirano, Periodic solutions for forced van der Pol type equations, Math. Econ. 1264 (2001), pp. $159-172$.

[9] R.D. Euzébio and J. Llibre, Periodic solutions of El Ni no model through the Vallis differential system, Discrete Contin. Dyn. Syst. Ser. A 34 (2014), pp. 3455-3469.

[10] T.H. Fay, The forced Van der Pol equation, Int. J. Math. Educ. Sci. Techol. 40 (2009), pp. 669-677.

[11] J. Guchkenheimer, K. Hoffman, and W. Weckesser, The forced Van der Pol equation I: The slow flow and its bifurcations, SIAM J. Appl. Dyn. Syst. 2 (2003), pp. 1-35.

[12] F.M.M. Kakmeni, S. Bowong, C. Tochawoua, and E. Kaptouom, Strange attractors and chaos control in a DuffingVan der Pol oscillator with two external periodic forces, J. Sound Vib. 227 (2004), pp. 783-799.

[13] F.M.M. Kakmeni, S. Bowong, C. Tchawoua, and E. Kaptouom, Chaos control and synchronization of a $\phi^{6}$-Van der Pol oscillator, Phys. Lett. A 322 (2004), pp. 305-323.

[14] H.K. Leung, Synchronization dynamics of coupled van der Pol systems, Phys. A 321 (2003), pp. 248-255.

[15] H. Li, Gröbner Bases in Ring Theory, World Scientific Publishing, Hackensack, NJ, 2011.

[16] Z.-G. Li, W. Xu, and X.-Y. Zhang, Analysis of chaotic behavior in the extended Duffing-Van der Pol system subject to additive non-symmetry biharmonical excitation, Appl. Math. Comput. 183 (2006), pp. 858-871.

[17] D. Liu and H. Yamaura, Chaos control of a $\phi^{6}$-Van der Pol oscillator driven by external excitation, Nonlinear Dyn. 68 (2012), pp. 95-105.

[18] J. Llibre and C. Vidal, Periodic solutions of a periodic FitzHugh-Nagumo differential system, preprint (2014).

[19] M. Ma, J. Zhou, and J. Cai, Practical synchronization of second-order nonautonomous systems with parameter mismatch and its applications, Nonlinear Dyn. 69 (2012), pp. 1285-1292.

[20] I.G. Malkin, Some Problems of the Theory of Nonlinear Oscillations (Russian), Gosudarstv. Izdat. Tehn.-Teor. Lit., Moscow, 1956.

[21] M. Roseau, Vibrations non linéaires et théorie de la stabilité (French), Springer Tracts in Natural Philosophy, Vol. 8, Springer-Verlag, Berlin-New York, 1966.

[22] H. Salarieh and A. Alasty, Control of stochastic chaos using sliding mode method, J. Comput. Appl. Math. 225 (2009), pp. 135-145.

[23] J.A. Sanders, F. VerhlstT, and J. Murdock, Averaging Method in Nonlinear Dynamical Systems, Applied Mathematical Science, Vol. 59, Springer, New York, 2007.

[24] M.S. Siewe, F.M.M. Kakmeni, and C. Tchawoua, Resonant oscillation and homoclinic bifurcation in a $\phi^{6}$-Van der Pol oscillator, Chaos Solitons and Fractals 21 (2004), pp. 841-853.

[25] W. Szemplinska-Stupnicka and J. Rudowski, Neimark bifurcation, almost-periodicity and chaos in the forced van der Pol-Duffing system in the neighbourhood of the principal resonance, Phys. Lett. A 192 (1994), pp. 201-206.

[26] R. Tchoukuegno, B.R.N. Nbendjo, and P. Woafo, Resonant oscillations and fractal basin boundaries of a particle in a $\phi^{6}$-potential, Phys. A 304 (2002), pp. 362-378.

[27] Y. Ueda and N. Akamatsu, Chaotically transitional phenomena in the forced negative-resistance oscillator, IEEE Trans. Circuits Syst. 28 (1981), pp. 217-224.

[28] A. Venkatesan and M. Lakshmanan, Bifurcation and chaos in the double-well Duffing-Van der Pol oscillator: Numerical and analytical studies, Phys. Rev. E 56 (1997), pp. 6321-6330.

[29] L. Yang, Recent advances on determining the number of real roots of parametric polynomials, J. Symbolic Comput. 28 (1999), pp. 225-242. 
[30] X. Yang, W. Xu, and Z. Sun, Effect of Gaussian white noise on the dynamical behaviours of an extended Duffing-Van der Pol oscillator, Int. J. Bifur. Chaos Appl. Sci. Eng. 16 (2006), pp. 2587-2600.

[31] X. Yang, W. Xu, and Z. Sun, Effect of bounded noise on the chaotic motion of a Duffing Van der pol oscillator in a $\phi^{6}$ potential, Chaos Solitons Fractals 27 (2006), pp. 778-788.

\section{Appendix. Root classification for a quintic polynomial}

In this section, we present a brief summary of the results about the number and multiplicities of the real/complex roots for a quintic polynomial with arbitrary coefficients presented in [29]. Indeed, consider the polynomial $P(x)=x^{5}+p x^{3}+$ $q x^{2}+u x+v$. So, the following table gives the number of real and complex roots and multiplicities of roots of $P(x)$ in all cases:$$
D_{5}>0 \wedge D_{4}>0 \wedge D_{3}>0 \wedge D_{2}>0
$$

$$
D_{5}>0 \wedge\left(D_{4} \leq 0 \vee D_{3} \leq 0 \vee D_{2} \leq 0\right)
$$
(10) $D_{5}=0 \wedge D_{4}=0 \wedge D_{3}=0 \wedge D_{2} \neq 0 \wedge F_{2} \neq 0$
(11) $D_{5}=0 \wedge D_{4}=0 \wedge D_{3}=0 \wedge D_{2} \neq 0 \wedge F_{2}=0$
(12) $\quad D_{5}=0 \wedge D_{4}=0 \wedge D_{3}=0 \wedge D_{2}=0$

where

$$
\begin{aligned}
D_{2}= & -p \\
D_{3}= & -12 p^{3}-45 q^{2}+40 p u \\
D_{4}= & -4 p^{3} q^{2}-27 q^{4}+12 p^{4} u+117 p q^{2} u-88 p^{2} u^{2}+160 u^{3}-40 p^{2} q v-300 q u v+125 p v^{2}, \\
D_{5}= & -4 p^{3} q^{2} u^{2}-27 q^{4} u^{2}+16 p^{4} u^{3}+144 p q^{2} u^{3}-128 p^{2} u^{4}+256 u^{5} \\
& +16 p^{3} q^{3} v+108 q^{5} v-72 p^{4} q u v-630 p q^{3} u v+560 p^{2} q u^{2} v-1600 q u^{3} v \\
& +108 p^{5} v^{2}+825 p^{2} q^{2} v^{2}-900 p^{3} u v^{2}+2250 q^{2} u v^{2}+2000 p u^{2} v^{2}-3750 p v^{3}+3125 v^{4}, \\
E_{2}= & 16 p^{4} q^{2}-48 p^{5} u+60 p^{2} q^{2} u+160 p^{3} u^{2}+900 q^{2} u^{2}-1100 p^{3} q v \\
& -3375 q^{3} v+1500 p q u v+625 p^{2} v^{2}, \\
F_{2}= & 3 q^{2}-8 p u .
\end{aligned}
$$

The polynomials $D_{i}, i=2,3,4,5, E_{2}$ and $F_{2}$ form a discriminant system which is sufficient for the classification of roots of the polynomial $P(x)$, which is described by the right column of the table. For instance, $\{1,1,1\}$ means three real simple roots and a pair of complex roots, and $\{3,1,1\}$ means a real triple root plus two real simple roots. 